\title{
Self-affine measures and vector-valued representations
}

\author{
by \\ QI-Rong Deng (Fuzhou and Hong Kong), \\ Xing-Gang He (Wuhan) and Ka-Sing LaU (Hong Kong)
}

\begin{abstract}
Let $A$ be a $d \times d$ integral expanding matrix and let $S_{j}(x)=A^{-1}\left(x+d_{j}\right)$ for some $d_{j} \in \mathbb{Z}^{d}, j=1, \ldots, m$. The iterated function system (IFS) $\left\{S_{j}\right\}_{j=1}^{m}$ generates self-affine measures and scale functions. In general this IFS has overlaps, and it is well known that in many special cases the analysis of such measures or functions is facilitated by expressing them in vector-valued forms with respect to another IFS that satisfies the open set condition. In this paper we prove a general theorem on such representation. The proof is constructive; it depends on using a tiling IFS $\left\{\psi_{j}\right\}_{j=1}^{l}$ to obtain a graph directed system, together with the associated probability on the vertices to form some transition matrices. As applications, we study the dimension and Lebesgue measure of a self-affine set, the $L^{q}$-spectrum of a self-similar measure, and the existence of a scaling function (i.e., an $L^{1}$-solution of the refinement equation).
\end{abstract}

1. Introduction. Throughout this paper we assume that $A$ is a $d \times d$ integral expanding matrix (i.e., all its eigenvalues have moduli $>1$ ) and $\mathcal{D}=\left\{d_{1}, \ldots, d_{m}\right\} \subset \mathbb{Z}^{d}$. We call $(A, \mathcal{D})$ an integral affine pair. This pair defines an iterated function system (IFS) $\left\{S_{j}\right\}_{j=1}^{m}$ on $\mathbb{R}^{d}$ by

$$
S_{j}(x)=A^{-1}\left(x+d_{j}\right), \quad x \in \mathbb{R}^{d} .
$$

It is known that under a suitable norm on $\mathbb{R}^{d}$, the expanding property of $A$ implies that the $S_{j}$ 's are contractive, hence there exists a unique nonempty compact set $K$ satisfying

$$
K=\bigcup_{j=1}^{m} S_{j}(K) .
$$

Alternatively, $K$ can be written in the form of radix expressions

$$
\left\{\sum_{n=1}^{\infty} A^{-n} d_{j_{n}}: d_{j_{n}} \in \mathcal{D}\right\} \text {. }
$$

2000 Mathematics Subject Classification: Primary 52C20, 52C22; Secondary 28A80.

Key words and phrases: digit set, Hausdorff dimension, $L^{q}$-spectrum, tile, scaling function, self-affine, vector-valued measure.

The research is partially supported by a HK RGC grant. 
We call the attractor $K$ a self-affine set, and a self-affine region if $K^{\circ} \neq \emptyset$. In the case where $|\operatorname{det} A|=m$, a self-affine region $K$ will tile $\mathbb{R}^{d}$ by certain translations of $K$ (cf., e.g., [19]); we call such a $K$ a self-affine tile. If we associate to the family $\left\{S_{j}\right\}_{j=1}^{m}$ a set of positive probability weights $\left\{p_{j}\right\}_{j=1}^{m}$, then there exists a unique probability measure $\mu$ supported on $K$ satisfying

$$
\mu(E)=\sum_{j=1}^{m} p_{j} \mu\left(S_{j}^{-1}(E)\right)=\sum_{j=1}^{m} p_{j} \mu\left(A(E)-d_{j}\right)
$$

for any Borel subset $E$ of $\mathbb{R}^{d}$. This measure is called a self-affine measure. If, in addition, the matrix $A$ is a constant multiple of an orthonormal matrix, (i.e., $A$ is a similarity and $\left\{S_{j}\right\}_{j=1}^{m}$ are similitudes), then in the above terminology we replace self-affine by self-similar.

The above IFS also plays a special role in the refinement equation in wavelet theory:

$$
f(x)=\sum_{j=1}^{m} a_{j} f\left(A x-d_{j}\right), \quad x \in \mathbb{R}^{d},
$$

where $a_{j} \in \mathbb{R}$ and $\sum_{j=1}^{m} a_{j}=|\operatorname{det} A|$. An $L^{1}$-solution of this equation is called a scaling function. It can be seen that the Radon-Nikodym derivative of the $\mu$ in (1.1) satisfies the refinement equation.

One of the most basic assumptions in the study of iterated function systems is the open set condition (OSC): there exists a bounded open set $U$ such that

$$
S_{j}(U) \subset U \text { for each } j \quad \text { and } \quad S_{i}(U) \cap S_{j}(U)=\emptyset \quad \text { if } i \neq j .
$$

Under this condition the attractor $K$ can be identified with a symbolic space and the invariant measure $\mu$ can be identified with a product measure on the symbolic space; their geometric and analytic properties are well understood (see, for example, [8], [2], [29], [37]). However, there are many important cases where the OSC is not satisfied (we loosely say that the IFS has over$l a p)$, for example when $m(=\# \mathcal{D})>|\operatorname{det} A|$ in the above $\left\{S_{j}\right\}_{j=1}^{m}$. The overlapping IFS's have very complicated and rich structure; there are many attempts to study them by imposing various conditions such as the transversality condition [35], the weak separation condition ([17], [23], [26], [33]) and the finite type condition [30].

In this paper we consider a vector-valued representation of the self-affine measure $\mu$ through a new IFS that satisfies the OSC. This approach was first used by Daubechies and Lagarias [3,4] for the refinement equation (1.2) with $A=[2], \mathcal{D}=\{0, \ldots, m-1\}$. The vector form of the equation is

$$
F(x)=T_{0} F(2 x)+T_{1} F(2 x-1)
$$

where $F:[0,1] \rightarrow \mathbb{R}^{m-1}$ is defined by $F(x)=[f(x), \ldots, f(x+m-2)]^{t}$ and 
$T_{0}, T_{1}$ are $(m-1) \times(m-1)$ matrices determined by the coefficients $a_{j}$, they are called transfer matrices. This representation initiated the investigation of the joint spectral radius to prove the existence and regularity of scaling functions (e.g., [18], [16], [27]). Another attempt of vector-valued representation was due to Strichartz [36] and Lau and Ngai [23] for the Bernoulli convolution associated with the golden ratio; it was used to give an explicit formula for the $L^{q}$-spectrum and verify the multifractal formalism for $q>0$ in such case. Feng has made a further investigation for $q \leq 0$ and extended this to the Pisot numbers $[9,10]$.

Note that all the established cases are on $\mathbb{R}$. Here we will concentrate on integral self-affine measures on $\mathbb{R}^{d}$. We will use a tiling IFS (i.e., the attractor is a self-affine tile) to be the new IFS with OSC for the vectorvalued representation. Our main result is

THEOREM 1.1. For each self-affine measure $\mu$ generated by an integral affine pair $(A, \mathcal{D})$, there exists a self-affine $\mathbb{Z}^{d}$-tile $T$ such that, for the set $\mathcal{E}=\left\{e_{1}, \ldots, e_{N}\right\}=\left\{e \in \mathbb{Z}^{d}: K \cap\left(T^{\circ}+e\right) \neq \emptyset\right\}$, the vector-valued measure

$$
\boldsymbol{\mu}(E)=\left[\mu\left((E \cap T)+e_{1}\right), \ldots, \mu\left((E \cap T)+e_{N}\right)\right]^{t}
$$

satisfies

$$
\boldsymbol{\mu}(\cdot)=\sum_{i=1}^{l} W_{i} \boldsymbol{\mu}\left(\psi_{i}^{-1}(\cdot)\right),
$$

where $l=\left|\operatorname{det}\left(A^{n_{0}}\right)\right|$ for some $n_{0} \geq 1,\left\{\psi_{i}(x)=A^{-n_{0}}\left(x+c_{i}\right)\right\}_{i=1}^{l}$ is the associated integral IFS generating $T$, and $W_{i}=\left[W_{i}(u, v)\right], 1 \leq i \leq l$, are nonnegative $N \times N$ matrices satisfying: (i) $W:=\sum_{i=1}^{l} W_{i}$ is irreducible; (ii) $W$ is Markov, i.e., the column sums of $W$ are all 1.

The $\mathbb{Z}^{d}$-tile $T$ in the theorem means $T$ admits $\mathbb{Z}^{d}$ as a tiling set. The IFS $\left\{\psi_{i}\right\}_{i=1}^{l}$ corresponding to $T$ satisfies the OSC. One of the most important consequences of this representation is that

$$
\boldsymbol{\mu}\left(\psi_{\sigma}(T)\right)=W_{\sigma} \boldsymbol{\mu}(T),
$$

where $\sigma=\left(i_{1}, \ldots, i_{n}\right) \in \Sigma_{l}^{n}, \Sigma_{l}=\{1, \ldots, l\}, \psi_{\sigma}=\psi_{i_{1}} \circ \cdots \circ \psi_{i_{n}}$ and $W_{\sigma}=W_{i_{1}} \cdots W_{i_{n}}$. The family of $\left\{\psi_{\sigma}(T)\right\}$ generates the Borel sets and the product of the matrices determines the local property (see Corollary 3.5) of $\mu$.

In the theorem the tile $T$ is generated by $A$ (or $A^{n_{0}}$ for some $n_{0}$ ) and a suitable choice of the digit set $\mathcal{C} \in \mathbb{Z}^{d}$ (it has to meet the technical requirement that $\mu(\partial T+e)=0$ for all $e \in \mathbb{Z}^{d}$, see $\left.\S 2\right)$. The set $\mathcal{E}=\left\{e_{1}, \ldots, e_{N}\right\}$ is considered as a set of vertices, and an edge from $e_{u}$ to $e_{v}$ exists if there exist $c_{i} \in \mathcal{C}$ and $d_{j} \in \mathcal{D}$ such that

$$
c_{i}-d_{j}+A e_{u}=e_{v}
$$


(see (3.4), Lemma 3.1(iii) and Lemma 3.3). The associated weights of this graph-directed system are the $W_{i}=\left[w_{i}(u, v)\right]$ with $w_{i}(u, v)=p_{j}$ where the $j$ is determined by (1.5).

The theorem also holds for the refinement equation (1.2) with some obvious adjustments. With the vector form, all the known theory for the joint spectral radius will go through.

For a given pair $(A, \mathcal{D})$, it is in general difficult to determine whether the self-affine set $K$ is a self-affine region, which is a necessary condition for (1.2) to have an $L^{1}$-solution; in the case $\# \mathcal{D}=|\operatorname{det} A|$, the $K$ is a self-affine tile [19]. This question has been studied in some detail in [14] and an algorithm was given there (see also [30], [37] for self-affine tiles). We make use of the main theorem to give a unified and more satisfactory criterion as follows:

Theorem 1.2. Let $\left\{W_{i}\right\}_{i=1}^{l}$ be the transition matrices in Theorem 1.1 corresponding to $p_{j}=1 / m$. Then the following conditions are equivalent:

(i) $K$ is a self-affine region, i.e., $K^{\circ} \neq \emptyset$;

(ii) $K$ has positive Lebesgue measure;

(iii) $\left(W_{\sigma} \mathbf{1}\right)^{\sim} \neq 0$ for any $\sigma=\left(i_{1}, \ldots, i_{n}\right), 1 \leq i_{j} \leq l, n>0$, where $\widetilde{v}$ denotes the vector with 1 in the nonzero entries of $v$ and 0 elsewhere; 1 is the column vector with 1 in all entries.

Let $\mathcal{F}=\left\{\mathbf{1}=v_{1}, \ldots, v_{r}\right\}$ be the set of all distinct $\left(W_{\sigma} \mathbf{1}\right)^{\sim}$. It is easy to see that $r \leq 2^{N}$. Hence we can determine whether $K^{\circ} \neq \emptyset$ in at most $2^{N}$ steps. It is known that the Lebesgue measure of such a $K$ is a rational number [14], and is an integer if $K$ is a tile [19]. We prove

THEOREM 1.3. Let $K$ be a self-affine region generated by an integral affine pair. Then the Lebesgue measure of $K$ is given by

$$
\mathcal{L}(K)=\sum_{i=1}^{r} a_{i} \alpha\left(v_{i}\right)
$$

where $\alpha\left(v_{i}\right)$ is the number of nonzero entries of $v_{i} \in \mathcal{F}$ and $\left\{a_{i}\right\}_{i=1}^{r}$ is defined through the matrix $G=[G(s, t)]_{r \times r}$ with

$$
G(s, t)=l^{-1} \#\left\{i:\left(W_{i} v_{s}\right)^{\sim}=v_{t}\right\}, \quad 1 \leq s, t \leq r .
$$

The detailed definition of $\left\{a_{i}\right\}_{i=1}^{r}$ is given in Theorem 5.3. For the case $K^{\circ}=\emptyset$, we want to determine its dimension. As a consequence of Theorem 1.2 , we have $v_{r}=0$ in $\mathcal{F}$ (after rearrangement), and the matrix $G$ in Theorem 1.3 can be expressed as

$$
G=\left[\begin{array}{cc}
G_{1} & g \\
0 & 1
\end{array}\right] .
$$

THEOREM 1.4. Let $K$ be the self-similar set coming from a pair $(A, \mathcal{D})$, where $A$ is a similarity, and suppose that $K^{\circ}=\emptyset$. Then $\operatorname{dim}_{\mathrm{B}} K=\operatorname{dim}_{\mathrm{H}} K$ 
$=d-\log \lambda_{1} / \log \varrho<d$, where $\lambda_{1}$ is the maximal eigenvalue of $G_{1}$ and $\varrho$ is the contraction ratio of the IFS.

As another application of Theorem 1.1, we consider the multifractal structure of the self-similar measure in (1.1). Let

$$
\alpha(x)=\lim _{r \rightarrow 0} \frac{\log \mu\left(B_{r}(x)\right)}{\log r}
$$

be the local dimension of $\mu$ at $x$. Let $K_{\alpha}=\{x \in K: \alpha=\alpha(x)\}$. A classical heuristic principle called the multifractal formalism says that

$$
\operatorname{dim}_{\mathrm{H}} K_{\alpha}=\tau^{*}(\alpha)
$$

where $\tau^{*}(\alpha)$ is the Legendre transform of $\tau(q)$, the $L^{q}$-spectrum of $\mu$ (see $\S 6$ ). The validity of the formalism has to be considered in individual cases and depends on the differentiability of $\tau(q)$. For example, if the IFS consists of similitudes and satisfies the OSC, then there is an explicit expression of $\tau(q)$ and the formalism holds ([8], [2], [7]). For overlapping IFS, there were extensive investigations of the Bernoulli convolution associated with the golden ratio [23] and the Pisot numbers [10], the convolution of the Cantor measure ([28], [12]) and some other related self-similar measures ([34], [38]). In these cases some extraordinary phenomena were revealed when $q<0$. There was also a study of the scaling functions where the coefficients are allowed to be negative (e.g., [5]).

By using the vector representation in Theorem 1.1, the product of matrices in (1.4) and the results in [11] and [24], we have

THEOREM 1.5. Let $\mu$ be the self-similar measure associated with the integral similar pair $(A, \mathcal{D})$. Then

$$
\tau(q)=\lim _{n \rightarrow \infty} \frac{\log \sum_{|\sigma|=n}\left\|W_{\sigma}\right\|_{1}^{q}}{n \log \varrho}, \quad q>0,
$$

where $|\sigma|$ is the length of $\sigma$ and $\left\|W_{\sigma}\right\|_{1}$ is the sum of all entries of $W_{\sigma}$. Moreover, $\tau(q)$ is differentiable and the multifractal formalism holds for $q>0$.

We organize the paper as follows. In $\S 2$, we set up some notation and introduce an auxiliary tiling system. We prove Theorem 1.1 in $\S 3$; the analog for the scaling function is also described. The vector-valued measure in Theorem 1.1 is constructive; we illustrate the construction by some examples in $\S 4$. In $\S 5$, we use a special case of Theorem 1.1 to consider self-affine sets; Theorems 1.2-4 are proved there. Finally, in $\S 6$, we consider the multifractal structure of integral self-similar measures, and prove Theorem 1.5.

2. The auxiliary tiling system. Let $(A, \mathcal{D})$ be an integral affine pair as in the last section with $\mathcal{D}=\left\{d_{1}, \ldots, d_{m}\right\}$ and let $\left\{S_{j}\right\}_{j=1}^{m}$ be the associated self-affine IFS. We will use the following symbols throughout: 
$\Sigma_{m}=\{1, \ldots, m\}$ (or just $\Sigma$ if there is no confusion) and $\Sigma^{*}=\bigcup_{n \geq 0} \Sigma^{n}$. For any $J=j_{1} \ldots j_{n} \in \Sigma^{n}$, let $S_{J}=S_{j_{1}} \circ \cdots \circ S_{j_{n}}$ and

$$
d_{J}=d_{j_{n}}+A d_{j_{n-1}}+\cdots+A^{n-1} d_{j_{1}}, \quad \mathcal{D}_{n}=\mathcal{D}+A \mathcal{D}+\cdots+A^{n-1} \mathcal{D} .
$$

We call a compact set $T \subseteq \mathbb{R}^{d}$ a tile if there exists a discrete set $\mathcal{T}$ (tiling set) such that $\mathbb{R}^{d}=\bigcup_{z \in \mathcal{T}}(T+z)$ and $\left(T^{\circ}+z\right) \cap\left(T^{\circ}+z^{\prime}\right)=\emptyset$ for any two distinct $z, z^{\prime} \in \mathcal{T}$. If the tiling set can be chosen to be $\mathbb{Z}^{d}$, then we call $T$ a $\mathbb{Z}^{d}$-tile. It is known that if $T$ is a self-affine tile (i.e., the attractor of an integral affine pair $(A, \mathcal{C})$ ), then $T$ admits a $\mathbb{Z}^{d}$-tiling if and only if $\mathcal{L}(T)$ (the Lebesgue measure of $T$ ) is 1 ; in this case $\# \mathcal{C}=|\operatorname{det} A|=l$ and $\mathcal{C}$ is a complete residue set, i.e., the set of cosets $\{[d]: d \in \mathcal{C}\}$ equals $\mathbb{Z}^{d} / A \mathbb{Z}^{d}[21]$.

Using Corollary 5 and Theorem 1 of [39] (or Theorem 1.3 of [19]), we have the following lemma which guarantees the existence of a $\mathbb{Z}^{d}$-tile for a given $A$.

Lemma 2.1. For any integral expanding matrix $A$, there exists an integer $k>0$ and a digit set $\mathcal{C} \subseteq \mathbb{Z}^{d}$ with $\# \mathcal{C}=\left|\operatorname{det}\left(A^{k}\right)\right|$ such that $T:=T\left(A^{k}, \mathcal{C}\right)$ is a $\mathbb{Z}^{d}$-tile.

Indeed, according to [39], the $k$ can be chosen such that all eigenvalues of $A^{k}$ are greater than $3 \sqrt{d}$ in modulus. For such $k$, let $Q=\left\{A^{k} x: x=\right.$ $\left.\left[x_{1}, \ldots, x_{d}\right]^{t}, x_{i} \in(-1 / 2,1 / 2]\right\}$. Then $\mathcal{C}=Q \cap \mathbb{Z}^{d}$ satisfies the condition of Lemma 2.1. In the one- or two-dimensional cases, the bound $3 \sqrt{d}$ can be improved to 2 . We also remark that the $A^{k}, k \geq 1$, in the above lemma cannot be taken to be $A$ as there exist expanding integral matrices $A$ (with size $d>3)$ such that $T(A, \mathcal{C})$ is not a $\mathbb{Z}^{d}$-tile for any integral digit set $\mathcal{C}$ with $\# \mathcal{C}=|\operatorname{det}(A)|([25$, corrigendum/addendum] and [32]). So far, for an integral similarity matrix $A$, no example has been found for which we must choose $k>1$.

In the following we will introduce an auxiliary IFS $\left\{\psi_{i}\right\}_{i=1}^{l}$ such that the attractor $T$ is a $\mathbb{Z}^{d}$-tile; this system satisfies the open set condition automatically and we will reduce the self-affine measure $\mu$ to be a vectorvalued self-affine measure $\boldsymbol{\mu}$ of $\left\{\psi_{i}\right\}_{i=1}^{l}$ in the next section. First we state

LEMMA 2.2. Let $\left\{S_{j}\right\}_{j=1}^{m}$ be the IFS generated by the integral affine pair $(A, \mathcal{D})$, let $\mu$ be a self-affine measure, and let $K$ be the attractor of the IFS $\left\{S_{j}\right\}_{j=1}^{m}$. Let $T=T(A, \mathcal{C})$ be $a \mathbb{Z}^{d}$-tile and let $V=\bigcup\left\{T^{\circ}+z: \mu(T+z)>0\right.$, $\left.z \in \mathbb{Z}^{d}\right\}$. Then

(i) $V$ is a nonempty open set and is invariant with respect to $\left\{S_{j}\right\}_{j=1}^{m}$;

(ii) if $V \cap K \neq \emptyset$, then $\mu(\partial T+z)=0$ for all $z \in \mathbb{Z}^{d}$ ( $\partial T$ is the boundary of $T)$.

Consequently, $\mu$ is concentrated on either $\bigcup_{z \in \mathbb{Z}^{d}}\left(T^{\circ}+z\right)$ or $\bigcup_{z \in \mathbb{Z}^{d}}(\partial T+z)$. 
Proof. Set $S_{j}(x)=A^{-1}\left(x+d_{j}\right), d_{j} \in \mathcal{D}$. If $\mu(T+z)>0$, then $\mu\left(S_{j}(T+z)\right)$ $\geq p_{j} \mu(T+z)>0$. Since $T=T(A, \mathcal{C})$ is a $\mathbb{Z}^{d}$-tile, $\mathcal{C}$ is a complete residue set of $A$. Hence there exist $c_{i} \in \mathcal{C}$ and $e \in \mathbb{Z}^{d}$ such that $z+d_{j}=c_{i}+A e$ and

$$
\begin{aligned}
S_{j}(T+z) & =A^{-1}\left(T+z+d_{j}\right)=A^{-1}\left(T+c_{i}+A e\right) \\
& =A^{-1}\left(T+c_{i}\right)+e \subseteq T+e .
\end{aligned}
$$

Hence $\mu(T+e) \geq \mu\left(S_{j}(T+z)\right) \geq p_{j} \mu(T+z)>0$ and $S_{j}\left(T^{\circ}+z\right) \subseteq T^{\circ}+e \subseteq V$. It follows that $S_{j}(V) \subseteq V$ for all $j$. This proves (i).

To prove (ii), we assume $V \cap K \neq \emptyset$; then we can find $x_{0} \in V \cap K, \varepsilon>0$ and $J_{0} \in \Sigma^{k}$ such that $S_{J_{0}}(K) \subseteq B_{\varepsilon}\left(x_{0}\right) \subseteq V$. We rearrange the distinct $S_{J}$ 's, $J \in \Sigma^{k}$, as $\left\{\phi_{j}\right\}_{j=1}^{r}$ with $\phi_{1}=S_{J_{0}}$ and let $w_{j}=\sum_{S_{J}=\phi_{j}} p_{J}>0$. Then we have

(a) $\phi_{1}(K)=S_{J_{0}}(K) \subseteq V$ and $\phi_{j}(V) \subseteq V, j=1, \ldots, r$;

(b) $K=\bigcup_{j=1}^{r} \phi_{j}(K)$;

(c) $\mu(\cdot)=\sum_{j=1}^{r} w_{j} \mu\left(\phi_{j}^{-1}(\cdot)\right)$.

For this new IFS $\left\{\phi_{j}\right\}_{j=1}^{r}$, let $\widetilde{\Sigma}_{r}=\{2,3, \ldots, r\}$ and let

$$
E_{n}=\bigcup_{J \in \Sigma_{r}^{n} \backslash \widetilde{\Sigma}_{r}^{n}} \phi_{J}(K) .
$$

For any $J=j_{1} \cdots j_{n} \in \Sigma_{r}^{n} \backslash \widetilde{\Sigma}_{r}^{n}$, there is an $1 \leq s \leq n$ such that $j_{s}=1$. Note that $\phi_{j}(K) \subseteq K$ for all $j$, so it follows from (a) that

$$
\phi_{J}(K) \subseteq \phi_{j_{1} \cdots j_{s-1}}\left(\phi_{j_{s}}(K)\right) \subseteq \phi_{j_{1} \cdots j_{s-1}}(V) \subseteq V
$$

and hence $E_{n} \subseteq V$. Using (b) and (c), we have

$$
\begin{aligned}
1 & \geq \mu(V) \geq \mu\left(E_{n}\right)=\sum_{J \in \Sigma_{r}^{n}} w_{J} \mu\left(\phi_{J}^{-1}\left(E_{n}\right)\right) \\
& \geq \sum_{J \in \Sigma_{r}^{n} \backslash \widetilde{\Sigma}_{r}^{n}} w_{J} \mu\left(\phi_{J}^{-1}\left(E_{n}\right)\right) \geq \sum_{J \in \Sigma_{r}^{n} \backslash \widetilde{\Sigma}_{r}^{n}} w_{J} \mu\left(\phi_{J}^{-1}\left(\phi_{J}(K)\right)\right) \\
& =\sum_{J \in \Sigma_{r}^{n} \backslash \widetilde{\Sigma}_{r}^{n}} w_{J} \mu(K)=\sum_{J \in \Sigma_{r}^{n}} w_{J}-\sum_{J \in \widetilde{\Sigma}_{r}^{n}} w_{J} \\
& =\left(\sum_{j=1}^{r} w_{j}\right)^{n}-\left(\sum_{j=2}^{r} w_{j}\right)^{n}=1-\left(1-w_{1}\right)^{n} .
\end{aligned}
$$

Since $w_{1}>0$, we have $\left(1-w_{1}\right)^{n} \rightarrow 0$ as $n \rightarrow \infty$. Therefore $\mu(V)=1$ and $\mu(\bar{V})=\mu(K)=1$. Noting that $\partial V=\bigcup\left\{\partial T+z: \mu(T+z)>0, z \in \mathbb{Z}^{d}\right\}$, we have $\mu(\partial V)=\mu(\partial T+z)=0$ for all $z \in \mathbb{Z}^{d}$.

In view of the above lemma, we need to find a $\mathbb{Z}^{d}$-tile $T$ such that

$$
\mu(\partial T+z)=0 \quad \text { for all } z \in \mathbb{Z}^{d},
$$


or equivalently, $\left(T^{\circ}+z\right) \cap K \neq \emptyset$ for some $z \in \mathbb{Z}^{d}$. This can be achieved by a certain translation of the tile:

LEMma 2.3. Let $K$ be the attractor of the integral affine pair $(A, \mathcal{D})$ and let $T=T(A, \mathcal{C})$ be a $\mathbb{Z}^{d}$-tile. Then there are $k>0$ and $e \in \mathcal{C}_{k}$ such that $T_{k}:=T\left(A^{k}, \mathcal{C}_{k}-e\right)$ is also a $\mathbb{Z}^{d}$-tile and $K \cap\left(T_{k}^{\circ}+z\right) \neq \emptyset$ for some $z \in \mathbb{Z}^{d}$.

Proof. Let $B_{\delta}\left(x_{0}\right) \subseteq T^{\circ}$ and let $a \in \mathbb{N}^{d}$ be such that $x_{0} \in K+a$. Since $T$ is a tile, $a+z \in T$ for some $z \in \mathbb{Z}^{d}$; hence there exist $c_{i_{j}} \in \mathcal{C}$ such that $a+z=\sum_{j=1}^{\infty} A^{-j} c_{i_{j}}$ (recall that $T=\left\{\sum_{n=1}^{\infty} A^{-n} x_{n}: x_{n} \in \mathcal{C}\right\}$ ). Let $I$ be the identity matrix and let

$$
a_{k}=-z+\left(I-A^{-k}\right)^{-1} \sum_{j=1}^{k} A^{-j} c_{i_{j}} .
$$

Note that $A^{-k}$ converges to the zero matrix, hence $\lim _{k \rightarrow \infty} a_{k}=a$. Let $k$ be such that $a_{k} \in a+B_{\delta}(0)$. Then $\left(K+a_{k}\right) \cap T^{\circ} \neq \emptyset$. Let $e=A^{k} \sum_{j=1}^{k} A^{-j} c_{i_{j}}=$ $\sum_{j=1}^{k} A^{k-j} c_{i_{j}}$. We see that $e \in \mathcal{C}_{k}$ and

$$
\begin{aligned}
T_{k} & =T\left(A^{k}, \mathcal{C}_{k}-e\right)=T\left(A^{k}, \mathcal{C}_{k}\right)-\sum_{j=1}^{\infty} A^{-k j} e \\
& =T\left(A^{k}, \mathcal{C}_{k}\right)-\left(I-A^{-k}\right)^{-1} A^{-k} e=T-\left(a_{k}+z\right) .
\end{aligned}
$$

This implies $K \cap\left(T_{k}^{\circ}+z\right) \neq \emptyset$.

We can now give the main result in this section.

TheOREM 2.4. Let $(A, \mathcal{D})$ be an integral affine pair. Then there is $n_{0}>0$ and a digit set $\mathcal{C} \subseteq \mathbb{Z}^{d}$ with $\# \mathcal{C}=\left|\operatorname{det}\left(A^{n_{0}}\right)\right|$ such that

(i) $T=T\left(A^{n_{0}}, \mathcal{C}\right)$ is a $\mathbb{Z}^{d}$-tile;

(ii) for any self-affine measure $\mu$ associated with $(A, \mathcal{D})$,

$$
\mu\left(\bigcup_{z \in \mathbb{Z}^{d}}(\partial T+z)\right)=0 .
$$

Proof. Lemma 2.1 implies that there exists an integer $k>0$ and a digit set $\widetilde{\mathcal{C}}$ such that $T\left(A^{k}, \widetilde{\mathcal{C}}\right)$ is a $\mathbb{Z}^{d}$-tile. Lemma 2.3 shows that there exists an integer $r>0$ and an integral vector $e \in \widetilde{\mathcal{C}}_{r}$ such that $K \cap\left(\left(T\left(A^{k r}\right.\right.\right.$, $\left.\left.\left.\widetilde{\mathcal{C}_{r}}-e\right)\right)^{\circ}+z\right) \neq \emptyset$ for some $z \in \mathbb{Z}^{d}$. Let $n_{0}=k r$ and $\mathcal{C}=\widetilde{\mathcal{C}}_{r}-e$. Then $T\left(A^{n_{0}}, \mathcal{C}\right)$ is a $\mathbb{Z}^{d}$-tile and $\left(T\left(A^{n_{0}}, \mathcal{C}\right)^{\circ}+z\right) \cap K \neq \emptyset$ for some $z \in \mathbb{Z}^{d}$. The remaining assertion follows from Lemma 2.2 .

3. Vector-valued self-affine measures. We will prove Theorem 1.1 via several lemmas. For the $n_{0}$ and $\mathcal{C}$ defined in Theorem 2.4, if $n_{0}>1$, let $\left\{\phi_{j}\right\}_{j=1}^{r}$ be the distinct $S_{J}$ 's, $J \in \Sigma^{n_{0}}$, and $w_{j}=\sum\left\{p_{J}: J \in \Sigma_{m}^{n_{0}}, S_{J}=\phi_{j}\right\}$. 
Then $\mu$ satisfies

$$
\mu(\cdot)=\sum_{j=1}^{r} w_{j} \mu\left(\phi_{j}^{-1}(\cdot)\right) .
$$

We can therefore replace $\left\{S_{j}\right\}_{j=1}^{m}$ and the corresponding probability weights $\left\{p_{j}\right\}_{j=1}^{m}$ by the IFS $\left\{\phi_{j}\right\}_{j=1}^{r}$ and $\left\{w_{j}\right\}_{j=1}^{r}$, respectively. Hence, in order to prove Theorem 1.1, we can assume without loss of generality that $n_{0}=1 \mathrm{in}$ Theorem 2.4, i.e., we assume

(H) $\quad T=T(A, \mathcal{C})$ is a $\mathbb{Z}^{d}$-tile such that $\mu\left(\bigcup_{z \in \mathbb{Z}^{d}}(\partial T+z)\right)=0$.

This assumption ensures that, for any $z \in \mathbb{Z}^{d}, \mu(T+z)>0$ if and only if $K \cap\left(T^{\circ}+z\right) \neq \emptyset$.

Let $\psi_{i}(x)=A^{-1}\left(x+c_{i}\right)$ for some $c_{i} \in \mathcal{C}$. Since we have two IFS's and so two index sets, to avoid confusion we will use $I, J$ to denote the multi-indices in $\Sigma_{m}^{*}$, and $\sigma, \tau$ to denote those in $\Sigma_{l}^{*}(l=|\operatorname{det} A|)$. Note that $S_{I}(0)=A^{-n} d_{I}$ and $S_{I}(x)=A^{-n}\left(x+d_{I}\right)$. Since $S_{j}$ and $\psi_{i}$ are defined by the same matrix $A$, one can show directly that

$$
S_{I}^{-1} \psi_{\sigma}(x)=x+c_{\sigma}-d_{I} \quad \forall I \in \Sigma_{m}^{n}, \sigma \in \Sigma_{l}^{n},
$$

and

$$
\begin{aligned}
\mu\left(\psi_{\sigma}(T+e)\right) & =\sum_{I \in \Sigma_{m}^{n}} p_{I} \mu\left(S_{I}^{-1}\left(\psi_{\sigma}(T+e)\right)\right) \\
& =\sum_{I \in \Sigma_{m}^{n}} p_{I} \mu\left(T+e+c_{\sigma}-d_{I}\right) \quad \forall e \in \mathbb{Z}^{d}, \sigma \in \Sigma_{l}^{n} .
\end{aligned}
$$

The above reveals the basic relationship of $\left\{S_{j}\right\}_{j=1}^{m}$ and $\left\{\psi_{i}\right\}_{i=1}^{l}$ and we make use of this to form a weighted directed graph system. Let

$$
\mathcal{E}=\left\{e_{1}, \ldots, e_{N}\right\}=\left\{e \in \mathbb{Z}^{d}: K \cap\left(T^{\circ}+e\right) \neq \emptyset\right\}
$$

and

$$
\mathcal{B}_{n}=\left\{\psi_{\sigma}(T)+e_{u}: e_{u} \in \mathcal{E}, \sigma \in \Sigma_{l}^{n}\right\}, \quad n \geq 0 .
$$

Since $T$ is a $\mathbb{Z}^{d}$-tile by our assumption $(\mathrm{H})$, it is easy to prove

LEMma 3.1. With the above notations, we have

(i) for any $E, F \in \mathcal{B}_{n}, E^{\circ} \cap F^{\circ} \neq \emptyset$ if and only if $E=F$;

(ii) $\mathcal{B}_{n}$ is a partition (with overlaps at the boundary) of the union $\bigcup_{E \in \mathcal{B}_{n}} E(\supseteq K)$

(iii) if $z \in \mathbb{Z}^{d}$ and $E=A^{-n}(T+z)$, then $E \in \mathcal{B}_{n}$ if and only if there are unique $\sigma \in \Sigma_{l}^{n}$ and $e_{u} \in \mathcal{E}$ such that $c_{\sigma}+A^{n} e_{u}=z$;

(iv) $\left(T^{\circ}+e_{u}\right) \cap K \neq \emptyset$ for all $e_{u} \in \mathcal{E}$.

Lemma 3.2. For any $I \in \Sigma_{m}^{n}, J \in \Sigma_{m}^{k}, \sigma \in \Sigma_{l}^{n}, \tau \in \Sigma_{l}^{k}$ and $z \in \mathbb{Z}^{d}$,

$$
c_{\sigma \tau}-d_{I J}+A^{n+k} z \in \mathcal{E} \quad \text { implies } \quad c_{\sigma}-d_{I}+A^{n} z \in \mathcal{E} .
$$


Proof. Observe that $\psi_{\sigma}(T)+z=A^{-n}\left(T+c_{\sigma}+A^{n} z\right)$, so (3.1) implies

$$
S_{I}^{-1}\left(\psi_{\sigma}(T)+z\right)=T+c_{\sigma}-d_{I}+A^{n} z .
$$

Since $\mu\left(S_{I}^{-1}(E)\right)=\sum_{J^{\prime} \in \Sigma_{m}^{k}} p_{J^{\prime}} \mu\left(S_{I J^{\prime}}^{-1}(E)\right)$, we have

$$
\begin{aligned}
\mu\left(T+c_{\sigma}-d_{I}+A^{n} z\right) & =\mu\left(S_{I}^{-1}\left(\psi_{\sigma}(T)+z\right)\right) \geq \mu\left(S_{I}^{-1}\left(\psi_{\sigma \tau}(T)+z\right)\right) \\
& \geq p_{J} \mu\left(S_{I J}^{-1}\left(\psi_{\sigma \tau}(T)+z\right)\right) \\
& =p_{J} \mu\left(T+c_{\sigma \tau}-d_{I J}+A^{n+k} z\right)>0,
\end{aligned}
$$

and the lemma follows.

As a crucial step to reformulate the self-affine measure of $\left\{S_{j}\right\}_{j=1}^{m}$ in terms of the auxiliary IFS $\left\{\psi_{i}\right\}_{i=1}^{l}$, we have

Lemma 3.3. The family $\left\{A^{-n}(T+z): z \in \mathbb{Z}^{d}, n \geq 0\right\}$ generates the Borel subsets of $\mathbb{R}^{d}$, and for any $z \in \mathbb{Z}^{d}$,

$$
\mu\left(A^{-n}(T+z)\right)=\sum_{v=1}^{N}\left(\sum\left\{p_{I}: I \in \Sigma_{m}^{n}, z-d_{I}=e_{v}\right\}\right) \mu\left(T+e_{v}\right)
$$

for some $e_{v} \in \mathcal{E}$.

Proof. The first part is clear as $T$ is a tile with $\mathbb{Z}^{d}$ as a tiling set. For the identity we note that

$$
\begin{aligned}
\mu\left(A^{-n}(T+z)\right) & =\sum_{I \in \Sigma_{m}^{n}} p_{I} \mu\left(S_{I}^{-1}\left(A^{-n}(T+z)\right)\right) \\
& =\sum_{I \in \Sigma_{m}^{n}} p_{I} \mu\left(T-d_{I}+z\right) .
\end{aligned}
$$

By the definition of $\mathcal{E}, \mu\left(T-d_{I}+z\right)>0$ if and only if $z-d_{I}=e_{v}$ for some $e_{v} \in \mathcal{E}$. The lemma follows by replacing $z-d_{I}$ with $e_{v}$ in the above expression.

It follows from Lemma 3.1(iii) that we only need to consider those sets $A^{-n}(T+z)$ such that $z=c_{\sigma}+A^{n} e_{u} \in \mathcal{E}$. In view of the above lemma, we define $W_{i}=\left[w_{i}(u, v)\right]_{N \times N}, 1 \leq i \leq l$, by

$$
w_{i}(u, v)= \begin{cases}p_{j}, & c_{i}-d_{j}+A e_{u}=e_{v} \text { for some } j, \\ 0, & \text { otherwise. }\end{cases}
$$

Then we have

Lemma 3.4. For any $\sigma=\sigma_{1} \cdots \sigma_{n} \in \Sigma_{l}^{n}$, let $W_{\sigma}=\left[w_{\sigma}(u, v)\right]$ be the corresponding product matrix. Then

$$
w_{\sigma}(u, v)=\sum\left\{p_{I}: I \in \Sigma_{m}^{n}, c_{\sigma}-d_{I}+A^{n} e_{u}=e_{v}\right\} .
$$


Proof. We will use induction. The identity is obviously true for $n=1$ by the definition of $W_{i}$. Assume it is true when $n=k-1$. For $n=k$, let $\sigma=\tau r, \tau \in \Sigma_{l}^{k-1}, J j \in \Sigma_{m}^{k}$. By Lemma 3.2, $c_{\sigma}+A^{k} e_{u}-d_{J j} \in \mathcal{E}$ implies $c_{\tau}+A^{k-1} e_{u}-d_{J} \in \mathcal{E}$. Let $W_{\tau}=\left[a_{u, v}\right]_{N \times N}$. By the induction hypothesis we have

$$
a_{u, v}=\sum\left\{p_{I}: I \in \Sigma_{m}^{k-1}, c_{\tau}-d_{I}+A^{k-1} e_{u}=e_{v}\right\} .
$$

This implies that

$$
\begin{aligned}
w_{\sigma}(u, v)= & \sum_{t=1}^{N} a_{u, t} w_{r}(t, v) \\
= & \sum_{t=1}^{N}\left(\sum\left\{p_{I}: I \in \Sigma_{m}^{k-1}, c_{\tau}-d_{I}+A^{k-1} e_{u}=e_{t}\right\}\right) \\
& \cdot\left(\sum\left\{p_{i}: c_{r}-d_{i}+A e_{t}=e_{v}\right\}\right) \\
= & \sum_{t=1}^{N} \sum\left\{p_{I i}: I \in \Sigma_{m}^{k-1}, c_{\tau}-d_{I}+A^{k-1} e_{u}=e_{t}, c_{r}-d_{i}+A e_{t}=e_{v}\right\} \\
= & \sum\left\{p_{J}: J \in \Sigma_{m}^{k}, c_{\sigma}-d_{J}+A^{k} e_{u}=e_{v}\right\},
\end{aligned}
$$

(the last equality follows from Lemma 3.2).

Proof of Theorem 1.1. We have assumed in $(\mathrm{H})$ that $n_{0}=1$ in the statement of Theorem 2.4. Also we assume all $d_{j}$ in $\mathcal{D}$ are distinct, otherwise we can combine the corresponding $S_{j}$ and $p_{j}$ together. Let $V=\bigcup_{j=1}^{N}\left(T^{\circ}+e_{j}\right)$. Lemma 2.2 implies that $V$ is open and invariant with respect to $\left\{S_{j}\right\}_{j=1}^{m}$, and $\mu(\partial T+z)=0$ for all $z \in \mathbb{Z}^{d}$. For

$$
\boldsymbol{\mu}(E)=\left[\mu\left((E \cap T)+e_{1}\right), \ldots, \mu\left((E \cap T)+e_{N}\right)\right]^{t}
$$

we have, by the self-affine identity,

$$
\begin{aligned}
(\boldsymbol{\mu}(E))_{v} & =\mu\left((T \cap E)+e_{v}\right)=\sum_{i=1}^{m} p_{i} \mu\left(A(T \cap E)+A e_{v}-d_{i}\right) \\
& =\sum_{i=1}^{m} p_{i} \mu\left(\left(\bigcup_{j=1}^{l}\left(T+c_{j}\right) \cap A(E)\right)+A e_{v}-d_{i}\right) \\
& =\sum_{i=1}^{m} p_{i} \mu\left(\bigcup_{j=1}^{l}\left(T \cap\left(A(E)-c_{j}\right)+c_{j}\right)+A e_{v}-d_{i}\right) .
\end{aligned}
$$


Use $\mu(\partial T+z)=0,(3.4)$ and the fact that $T$ is a $\mathbb{Z}^{d}$-tile to obtain

$$
\begin{aligned}
(\boldsymbol{\mu}(E))_{v} & =\sum_{i=1}^{m} \sum_{j=1}^{l} p_{i} \mu\left(T \cap\left(A(E)-c_{j}\right)+c_{j}+A e_{v}-d_{i}\right) \\
& =\sum_{j=1}^{l} \sum_{r=1}^{N} w_{j}(v, r) \mu\left(T \cap\left(A(E)-c_{j}\right)+e_{r}\right) .
\end{aligned}
$$

This implies $\boldsymbol{\mu}(E)=\sum_{j=1}^{l} W_{j} \boldsymbol{\mu}\left(\psi_{j}^{-1}(E)\right)$.

To prove statement (i), we note that for any $1 \leq u, v \leq N$, our assumption $(\mathrm{H})$ on $T$ implies that $K \cap\left(T^{\circ}+e_{u}\right) \neq \emptyset$, so there exists an integer $n$ and $I \in \Sigma_{m}^{n}$ such that $S_{I}\left(T+e_{v}\right) \subseteq T+e_{u}$. Since

$$
S_{I}\left(T+e_{v}\right)=A^{-n}\left(T+e_{v}+d_{I}\right)
$$

with $e_{v}+d_{I} \in \mathbb{Z}^{d}$, Lemma 3.1(iii) implies that there exists $\sigma \in \Sigma_{l}^{n}$ such that $c_{\sigma}-d_{I}+A^{n} e_{u}=e_{v}$; by Lemma 3.4, we see that for $W=\sum_{i=1}^{m} W_{i}$, the $(u, v)$ entry of $W^{n}$ is $\geq p_{I}$ and hence is positive. Thus we have proved that $W$ is irreducible.

For (ii), we first consider the expression $c_{i}-d_{s}+A e_{u}=e_{v}$ in (3.4). We claim that the pair $\left(e_{u}, c_{i}\right)$ is uniquely determined by $e_{v}$ and $d_{s}$. Indeed, if $c_{j}-d_{s}+A e_{u^{\prime}}=e_{v}$, then $A^{-1}\left(c_{i}-c_{j}\right)=e_{u^{\prime}}-e_{u} \in \mathbb{Z}^{d}$. Since $T$ is a $\mathbb{Z}^{d}$-tile, $\left\{c_{1}, \ldots, c_{l}\right\}$ is a complete set of residues $(\bmod A)[21]$, and we conclude that $u^{\prime}=u$ and $i=j$, which yields the claim.

It follows from the claim that distinct pairs $\left(e_{u}, c_{i}\right)$ and $\left(e_{u^{\prime}}, c_{j}\right)$ with $w_{i}(u, v)>0$ and $w_{j}\left(u^{\prime}, v\right)>0$ correspond to distinct $d_{s}$. Hence

$$
\sum_{u=1}^{N} w(u, v)=\sum_{i=1}^{l} \sum_{u=1}^{N} w_{i}(u, v) \leq \sum_{s=1}^{m} p_{s}=1, \quad v=1, \ldots, N,
$$

i.e., the column sums of $W$ are $\leq 1$. On the other hand, by the vector self-affine identity just proved, $\left[\mu\left(T+e_{1}\right), \ldots, \mu\left(T+e_{N}\right)\right]^{t}$ is a positive 1eigenvector of $W$. This implies that all column sums of $W$ must be 1 . The proof is complete.

The above proof yields

COROLlary 3.5. With the same assumptions and notations of Theorem 1.1, we have

$$
\boldsymbol{\mu}\left(\psi_{\sigma}(T)\right)=W_{\sigma} \boldsymbol{\mu}(T), \quad \forall \sigma \in \Sigma^{*} .
$$

We remark that in the above proof, each $p_{j}$ appears exactly once in each column of $W$. Also the matrices $\left\{W_{i}\right\}_{i=1}^{l}$ are not unique, not even the same size. They depend on the choice of $\mathcal{C}$ for the tile $T$; an example is given in Section 4 for the case $A=[3]$ on $\mathbb{R}$. 
For the actual construction of $\boldsymbol{\mu}$ and $W_{i}$, we have to find the set $\mathcal{E}$ in the theorem as both the tile $T$ and the attractor $K$ may not be expressed explicitly. We provide an algorithm to construct $\mathcal{E}$ by using the expression in (3.4).

Proposition 3.6. Let $K$ be the attractor of $(A, \mathcal{D})$, and let $T=T(A, \mathcal{C})$ be a $\mathbb{Z}^{d}$-tile such that $K \cap\left(T^{\circ}+z\right) \neq \emptyset$ for some $z \in \mathbb{Z}^{d}$ as above. Let $\mathcal{E}_{0}=\emptyset$ and let $\emptyset \neq \mathcal{E}_{1} \subseteq \mathcal{E}$. Define

$$
\mathcal{E}_{n+1}=\mathcal{E}_{n} \cup\left(\mathbb{Z}^{d} \cap A^{-1}\left(\left(\mathcal{E}_{n} \backslash \mathcal{E}_{n-1}\right)+\mathcal{D}-\mathcal{C}\right)\right), \quad n>0 .
$$

Then there is an $n>0$ such that $\mathcal{E}_{n}=\mathcal{E}_{n+1}$, and for this $n$ we have $\mathcal{E}_{n}=\mathcal{E}$.

Proof. Since $\mathcal{E}$ is a finite set, we need only prove $\mathcal{E}_{n} \subseteq \mathcal{E}$ for all $n>0$, and $\mathcal{E} \subseteq \bigcup_{n>0} \mathcal{E}_{n}$.

We prove the first inclusion by induction. Assume that $\mathcal{E}_{n} \subseteq \mathcal{E}$ and let $z \in \mathcal{E}_{n+1} \backslash \mathcal{E}_{n}$. Then there exist $e \in \mathcal{E}_{n}, d_{i} \in \mathcal{D}$ and $c_{j} \in \mathcal{C}$ such that $z=A^{-1}\left(e+d_{i}-c_{j}\right)$. Hence

$$
\mu(T+z) \geq p_{i} \mu\left(A(T+z)-d_{i}\right)=p_{i} \mu\left(A T+e-c_{j}\right) .
$$

Note that $A T \supseteq T+c_{j}$ and $e \in \mathcal{E}_{n} \subseteq \mathcal{E}$, so $\mu(T+z) \geq p_{i} \mu(T+e)>0$. This implies $\mathcal{E}_{n+1} \subseteq \mathcal{E}$ and induction follows.

For the second inclusion, let $e_{v} \in \mathcal{E}$. Choose $e_{u} \in \mathcal{E}_{1}$. Since $W=\sum_{i=1}^{l} W_{i}$ is irreducible, there exist $e_{v_{1}}, \ldots, e_{v_{n}} \in \mathcal{E}$ with $v_{1}=u$ and $v_{n}=v$ such that $w\left(v_{j+1}, v_{j}\right)>0$. From the definition of $\left\{W_{i}\right\}_{i=1}^{l}$, we see that $e_{v_{j+1}} \in$ $A^{-1}\left(e_{v_{j}}+\mathcal{D}-\mathcal{C}\right)$. Therefore $e_{v} \in \mathcal{E}_{n}$ from the definition of $\mathcal{E}_{j}$ and since $e_{v_{1}} \in \mathcal{E}_{1}$. Hence $\mathcal{E} \subseteq \bigcup_{n>0} \mathcal{E}_{n}$.

In the next section, we illustrate this algorithm by some examples. To conclude this section, we consider the refinement equation

$$
f(x)=\sum_{j=1}^{m} a_{j} f\left(A x-d_{j}\right), \quad x \in \mathbb{R}^{d},
$$

where $A$ and $d_{j} \in \mathcal{D}$ are as before, and the coefficients $\left\{a_{j}\right\}_{j=1}^{m}$ are real and satisfy $\sum_{j=1}^{m} a_{j}=|\operatorname{det} A|$. The $L^{1}$-solution of the equation is called a scaling function. In this case, $f$ is supported by $K$ and is unique up to a constant multiple. It is well known that for the scaling function in $\mathbb{R}$ with scaling 2 , the analysis depends very much on a vector-valued setup ([3], [4], [16], $[27])$. For the higher dimensional case, the same technique in the proof of Theorem 1.1 can be used for the vector-valued reduction. Below we state such a theorem without proof.

Similarly to the definition of $W_{i}$, we define the $N \times N$ matrices $C_{i}, 1 \leq$ $i \leq l$, by

$$
C_{i}(u, v)= \begin{cases}a_{j}, & c_{i}-d_{j}+A e_{u}=e_{v} \text { for some } j \\ 0, & \text { otherwise }\end{cases}
$$


Then $l=|\operatorname{det} A|$ is an eigenvalue of $C:=\sum_{j=1}^{l} C_{j}$. For any function $f$, we define an $N$-dimensional vector function $F=\left(F_{1}, \ldots, F_{N}\right)$ by

$$
F_{i}(x)= \begin{cases}f\left(x+e_{i}\right), & x \in T, \\ 0, & \text { otherwise. }\end{cases}
$$

THEOREM 3.7. Let $f$ be a function supported by $K$, the attractor of $(A, \mathcal{D})$, and let $F$ be defined as above. Then $f$ is an $L^{1}$-solution of the refinement equation (3.8) if and only if $F$ is an $L^{1}$-solution of

$$
F(x)=\sum_{j=1}^{l} C_{j} F \circ \psi_{j}^{-1}(x), \quad \text { a.e. } x \in \mathbb{R}^{d} .
$$

Remark. Since $\partial T$ has Lebesgue measure zero and $f$ is defined a.e. with respect to Lebesgue measure, the technicality on $\mu(\partial T+z)=0$ in the proof of Theorem 1.1 is not needed (as $\mu$ is absolutely continuous and $\mu\left(\bigcup_{z \in \mathbb{Z}^{d}}(\partial T+z)\right)=0$ automatically). Hence we do not need Lemmas 2.2 and 2.3 in the proof.

There is vast literature on scaling functions on $\mathbb{R}$ using the joint spectral radius associated with the above $\left\{C_{i}\right\}_{i=1}^{l}$. Most of the theorems can be generalized directly once the vector-valued form is established. We list one of these as an example ([27], [17]). For any vector $\nu \in \mathbb{R}^{N}$, let $H(\nu)$ be the linear subspace spanned by $\left\{C_{\sigma}\left(I-C_{i}\right) \nu: i=1, \ldots, l, \sigma \in \Sigma_{l}^{*}\right\}$, where $I$ is the $N \times N$ identity matrix.

Proposition 3.8. With the above notations, let $\nu$ be a nonzerol-eigenvector of $\sum_{j=1}^{l} C_{j}$. Then the following three statements are equivalent:

(i) the equation (3.8) has a nontrivial $L^{1}$-solution;

(ii) $\lim _{n \rightarrow \infty} l^{-n} \sum_{\sigma \in \Sigma_{l}^{n}} \sum_{j=1}^{l}\left\|C_{\sigma}\left(I-C_{j}\right) \nu\right\|=0$;

(iii) there exists an integer $k>0$ such that

$$
l^{-k} \sum_{\sigma \in \Sigma_{l}^{k}}\left\|C_{\sigma} w\right\|<1 \quad \forall w \in H(\nu),\|w\| \leq 1 .
$$

4. Examples. In this section, we will illustrate the construction of the vector form in Theorem 1.1. First we consider the well known cases associated with $A=2$ on $\mathbb{R}$ under our present setting.

ExAmPle 4.1. Let $A=2, \mathcal{D}=\{0, \ldots, m-1\}$ and let $\mu$ be the self-similar measure generated by $(A, \mathcal{D})$ with associated weights $\left\{p_{j}\right\}_{j=1}^{m}$.

The attractor is $K=[0, m-1]$. According to Theorem 1.1, we choose $\mathcal{C}=\{0,1\}$; then $T=[0,1]$. It follows that

$$
\mathcal{E}=\{i: \mu(K \cap[i, i+1])>0\}=\{0,1, \ldots, m-2\} .
$$


Let $c_{i}=i-1, d_{j}=j-1$ and $e_{u}=u-1 \in \mathcal{E}$. Then the definition of $W_{i}$ in (3.4) implies that $w_{i}(u, v)=p_{j}$ if and only if $j=2 u-v+i-1$. Hence

$$
\begin{gathered}
W_{1}=\left[p_{2 u-v}\right]=\left[\begin{array}{ccccc}
p_{1} & 0 & 0 & \cdots & 0 \\
p_{3} & p_{2} & p_{1} & \cdots & 0 \\
p_{5} & p_{4} & p_{3} & \cdots & 0 \\
\vdots & \vdots & \vdots & \ddots & \vdots \\
0 & 0 & 0 & \cdots & p_{m-1}
\end{array}\right], \\
W_{2}=\left[p_{2 u-v+1}\right]=\left[\begin{array}{ccccc}
p_{2} & p_{1} & 0 & \cdots & 0 \\
p_{4} & p_{3} & p_{2} & \cdots & 0 \\
p_{6} & p_{5} & p_{4} & \cdots & 0 \\
\vdots & \vdots & \vdots & \ddots & \vdots \\
0 & 0 & 0 & \cdots & p_{m}
\end{array}\right] .
\end{gathered}
$$

The $W_{1}$ and $W_{2}$ are uniquely determined regardless of the choice of $\mathcal{C}$, since, for any other digit set $\mathcal{C}^{\prime}$ such that $T\left(2, \mathcal{C}^{\prime}\right)$ is a $\mathbb{Z}$-tile of $\mathbb{R}$, there is an integer $k$ such that $T(2, \mathcal{C})=T\left(2, \mathcal{C}^{\prime}\right)+k$ and the sets $\left\{T+e_{i}\right\}$ are unchanged.

EXAmPle 4.2. Let $A=3, \mathcal{D}=\{0,2,4,6\}$ and let $\mu$ be the self-similar measure generated by $(A, \mathcal{D})$ with associated weights $\left\{p_{j}\right\}_{j=1}^{4}$.

The attractor is $K=[0,3]$. If we choose $\mathcal{C}=\{0,1,2\}$, then $T=[0,1]$ and hence $\mathcal{E}=\{0,1,2\}$. Let

$$
c_{i}=i-1, \quad d_{j}=2 j-2, \quad e_{u}=u-1 .
$$

Then the definition of $W_{i}$ implies that $w_{i}(u, v)=p_{j}$ if and only if $2 j=$ $3 u-v+i-1$. Hence we have

$$
W_{1}=\left[\begin{array}{ccc}
p_{1} & 0 & 0 \\
0 & p_{2} & 0 \\
p_{4} & 0 & p_{3}
\end{array}\right], \quad W_{2}=\left[\begin{array}{ccc}
0 & p_{1} & 0 \\
p_{3} & 0 & p_{2} \\
0 & p_{4} & 0
\end{array}\right], \quad W_{3}=\left[\begin{array}{ccc}
p_{2} & 0 & p_{1} \\
0 & p_{3} & 0 \\
0 & 0 & p_{4}
\end{array}\right] .
$$

These coincide with the $T_{0}, T_{1}$ and $T_{2}$ defined in [28].

If we choose $\mathcal{C}=\{-1,0,1\}$, then $T=[-1 / 2,1 / 2]$ and so $\mathcal{E}=\{0,1,2,3\}$. For this choice,

$$
W_{1}=\left[\begin{array}{cccc}
0 & 0 & 0 & 0 \\
p_{2} & 0 & p_{1} & 0 \\
0 & p_{3} & 0 & p_{2} \\
0 & 0 & p_{4} & 0
\end{array}\right], \quad W_{2}=\left[\begin{array}{cccc}
p_{1} & 0 & 0 & 0 \\
0 & p_{2} & 0 & p_{1} \\
p_{4} & 0 & p_{3} & 0 \\
0 & 0 & 0 & p_{4}
\end{array}\right], \quad W_{3}=\left[\begin{array}{cccc}
0 & p_{1} & 0 & 0 \\
p_{3} & 0 & p_{2} & 0 \\
0 & p_{4} & 0 & p_{3} \\
0 & 0 & 0 & 0
\end{array}\right] .
$$

We see that, unlike the case in Example 4.1, if we choose a different $\mathcal{C}$ (and hence $T$ ), we may have different $\mathcal{E}$ and $W_{j}$. 
Also, note that $\mathcal{D} \subset 2 \mathbb{Z}$; if we consider $\nu(E) \doteq \mu(2 E)$ and choose $\mathcal{C}=$ $\{0,1,2\}$, let $\boldsymbol{\mu}(E)=(\mu(E \cap(2 T)), \mu(E \cap(2 T)+2))^{t}$. Then

$$
\boldsymbol{\mu}(E)=\sum_{j=1}^{3} W_{j} \boldsymbol{\mu}(3 E-2 j)
$$

with

$$
W_{1}=\left[\begin{array}{cc}
p_{1} & 0 \\
p_{4} & p_{3}
\end{array}\right], \quad W_{2}=\left[\begin{array}{cc}
p_{2} & p_{1} \\
0 & p_{4}
\end{array}\right], \quad W_{3}=\left[\begin{array}{cc}
p_{3} & p_{2} \\
0 & 0
\end{array}\right]
$$

This is simpler than the previous two representations.

ExAmple 4.3. Let $A=\left[\begin{array}{cc}1 & 1 \\ -1 & 1\end{array}\right], \mathcal{D}=\left\{[0,0]^{t},[1,0]^{t},[0,1]^{t}\right\}$ and let $\mu$ be the self-similar measure generated by $(A, \mathcal{D})$ with associated weights $\left\{p_{j}\right\}_{j=1}^{3}$ and $K$ be the attractor.

Choose $\mathcal{C}=\left\{[0,0]^{t},[1,0]^{t}\right\}$. Then $T=T(A, \mathcal{C})$ is a $\mathbb{Z}^{2}$-tile (the twin dragon). For this example, both $K$ and $T$ are more complicated. Note that $T \subseteq K$ and $\mathcal{D}-\mathcal{C}=\left\{[0,-1]^{t},[1,-1]^{t},[0,0]^{t},[1,0]^{t},[0,1]^{t}\right\}$. Let $\mathcal{E}_{1}=\left\{[0,0]^{t}\right\}$. By Proposition 3.6 we find $\mathcal{E}_{i}$ inductively and the process stops at the 11 th step with

$$
\begin{gathered}
\mathcal{E}_{11}=\left\{[-1,-2]^{t},[-2,-1]^{t},[-2,0]^{t},[0,-2]^{t},[-1,-1]^{t},[-1,0]^{t},[0,-1]^{t},\right. \\
\left.[0,0]^{t},[-1,1]^{t},[1,-1]^{t},[1,0]^{t},[0,1]^{t},[1,1]^{t}\right\} .
\end{gathered}
$$

Therefore $\mathcal{E}=\mathcal{E}_{11}$ and there are 13 translates of $T^{\circ}$ intersecting $K$. By the definition of $W_{j}$ in (3.4), we have

$$
W_{1}=\left[\begin{array}{ccccccccccccc}
0 & 0 & 0 & 0 & 0 & 0 & 0 & 0 & 0 & 0 & 0 & 0 & 0 \\
0 & 0 & 0 & 0 & 0 & 0 & 0 & 0 & 0 & 0 & 0 & 0 & 0 \\
0 & 0 & 0 & 0 & 0 & 0 & 0 & 0 & 0 & 0 & 0 & 0 & 0 \\
0 & 0 & 0 & 0 & 0 & 0 & 0 & 0 & 0 & 0 & 0 & 0 & 0 \\
0 & p_{3} & p_{1} & 0 & 0 & 0 & 0 & 0 & 0 & 0 & 0 & 0 & 0 \\
0 & 0 & 0 & 0 & 0 & p_{3} & 0 & 0 & p_{1} & 0 & 0 & 0 & 0 \\
p_{3} & p_{2} & 0 & 0 & p_{1} & 0 & 0 & 0 & 0 & 0 & 0 & 0 & 0 \\
0 & 0 & 0 & 0 & 0 & p_{2} & p_{3} & p_{1} & 0 & 0 & 0 & 0 & 0 \\
0 & 0 & 0 & 0 & 0 & 0 & 0 & 0 & 0 & 0 & 0 & p_{3} & 0 \\
p_{2} & 0 & 0 & p_{1} & 0 & 0 & 0 & 0 & 0 & 0 & 0 & 0 & 0 \\
0 & 0 & 0 & 0 & 0 & 0 & p_{2} & 0 & 0 & p_{1} & 0 & 0 & 0 \\
0 & 0 & 0 & 0 & 0 & 0 & 0 & 0 & 0 & 0 & p_{3} & p_{2} & p_{1} \\
0 & 0 & 0 & 0 & 0 & 0 & 0 & 0 & 0 & 0 & p_{2} & 0 & 0
\end{array}\right],
$$




$$
W_{2}=\left[\begin{array}{ccccccccccccc}
0 & p_{1} & 0 & 0 & 0 & 0 & 0 & 0 & 0 & 0 & 0 & 0 & 0 \\
0 & 0 & p_{3} & 0 & 0 & 0 & 0 & 0 & 0 & 0 & 0 & 0 & 0 \\
0 & 0 & 0 & 0 & 0 & 0 & 0 & 0 & p_{3} & 0 & 0 & 0 & 0 \\
p_{1} & 0 & 0 & 0 & 0 & 0 & 0 & 0 & 0 & 0 & 0 & 0 & 0 \\
0 & 0 & p_{2} & 0 & p_{3} & p_{1} & 0 & 0 & 0 & 0 & 0 & 0 & 0 \\
0 & 0 & 0 & 0 & 0 & 0 & 0 & p_{3} & p_{2} & 0 & 0 & p_{1} & 0 \\
0 & 0 & 0 & p_{3} & p_{2} & 0 & p_{1} & 0 & 0 & 0 & 0 & 0 & 0 \\
0 & 0 & 0 & 0 & 0 & 0 & 0 & p_{2} & 0 & p_{3} & p_{1} & 0 & 0 \\
0 & 0 & 0 & 0 & 0 & 0 & 0 & 0 & 0 & 0 & 0 & 0 & p_{3} \\
0 & 0 & 0 & p_{2} & 0 & 0 & 0 & 0 & 0 & 0 & 0 & 0 & 0 \\
0 & 0 & 0 & 0 & 0 & 0 & 0 & 0 & 0 & p_{2} & 0 & 0 & 0 \\
0 & 0 & 0 & 0 & 0 & 0 & 0 & 0 & 0 & 0 & 0 & 0 & p_{2} \\
0 & 0 & 0 & 0 & 0 & 0 & 0 & 0 & 0 & 0 & 0 & 0 & 0
\end{array}\right] .
$$

5. Application to self-affine sets. This section is devoted to the calculation of the Lebesgue measure and Hausdorff dimension of integral selfaffine sets. These problems have been investigated in [37] and [14]. We will make use of the matrix representation of Section 3 to give an alternative approach, which unifies the considerations with the measures and functions and seems to be simpler.

We will use the notations defined in the previous sections with the special set of probabilities $p_{1}=\cdots=p_{m}=1 / m$ (actually any set of positive probabilities $\left\{p_{i}\right\}_{i=1}^{l}$ will do). We also suppose that the assumption $(\mathrm{H})$ holds for the auxiliary affine system.

For any $r \times s$ matrix (or vector if $s=1$ ) $B=\left(b_{i j}\right)$, let $B^{\sim}=\left(\widetilde{b}_{i j}\right)$ be such that $\widetilde{b}_{i j}$ equals 1 if $b_{i j} \neq 0$, and equals 0 if $b_{i j}=0$. For any two nonnegative matrices $B$ and $C$ such that $B C$ is well defined, we have

$$
(B C)^{\sim}=\left(B C^{\sim}\right)^{\sim} \quad \forall B, C \geq 0 .
$$

This follows from the fact that $\sum_{s} b_{i s} c_{s j} \neq 0$ if and only if $\sum_{s} b_{i s} \widetilde{c}_{s j} \neq 0$.

We first provide a constructive way to check if $K$ is a self-affine region, i.e., $K^{\circ} \neq \emptyset$ (see the remark after the theorem). By Theorem 1.1, we have

TheOREM 5.1. Let $K$ be the attractor generated by the integral affine pair $(A, \mathcal{D})$ and let $T=T(A, \mathcal{C})$ be a $\mathbb{Z}^{d}$-tile satisfying $(\mathrm{H})$. Then the following statements are equivalent:

(i) $K$ is a self-affine region, i.e., $K^{\circ} \neq \emptyset$;

(ii) $\mathcal{L}(K)>0$;

(iii) $W_{\sigma} \neq 0$ (equivalently $\left(W_{\sigma} \mathbf{1}\right)^{\sim} \neq 0$ ) for any $\sigma \in \Sigma_{l}^{n}, n>0$; 
(iv) $T \subseteq \bigcup_{j=1}^{N}\left(K-e_{j}\right)$, where $\mathcal{E}=\left\{e_{1}, \ldots, e_{N}\right\}=\left\{e \in \mathbb{Z}^{d}: \mu(T+e)>0\right\}$ as in (3.3).

Proof. The implications (i) $\Rightarrow$ (ii) and (iv) $\Rightarrow$ (i) are obviously true.

(ii) $\Rightarrow$ (iii). If there exist $n>0$ and $\sigma \in \Sigma_{l}^{n}$ such that $W_{\sigma}=0$, then the identity in Theorem 1.1 implies that

$$
\boldsymbol{\mu}(\cdot)=\sum_{\tau \in \Sigma_{l}^{n} \backslash\{\sigma\}} W_{\tau} \boldsymbol{\mu}\left(\psi_{\tau}^{-1}(\cdot)\right) .
$$

It follows that $\boldsymbol{\mu}$ is supported by the attractor of the IFS $\left\{\psi_{\tau}: \tau \in \Sigma_{l}^{n} \backslash\{\sigma\}\right\}$ which is of Lebesgue measure zero. Since we have $\operatorname{supp} \boldsymbol{\mu}=\bigcup_{j=1}^{N} \operatorname{supp} \boldsymbol{\mu}_{j}=$ $\bigcup_{j=1}^{N}\left(T \cap\left(K-e_{j}\right)\right)$, it follows that $\mathcal{L}\left(T \cap\left(K-e_{j}\right)\right)=0$. Therefore $\mathcal{L}(K) \leq$ $\sum_{j=1}^{N} \mathcal{L}\left(\left(T+e_{j}\right) \cap K\right)=0$, a contradiction.

(iii) $\Rightarrow$ (iv). Assume that $T \nsubseteq \bigcup_{j=1}^{N}\left(K-e_{j}\right)$. Then $T^{\circ} \backslash \bigcup_{j=1}^{N}\left(K-e_{j}\right)$ is a nonempty open set. Since $\mu$ is supported by $K$, the definition of $\boldsymbol{\mu}$ implies that $\boldsymbol{\mu}$ is supported by $\bigcup_{j=1}^{N}\left(K-e_{j}\right)$, hence $\boldsymbol{\mu}\left(T^{\circ} \backslash \bigcup_{j=1}^{N}\left(K-e_{j}\right)\right)=0$. Since $T$ is the attractor of $\left\{\psi_{j}\right\}_{j=1}^{l}$, there exist $n>0$ and $\sigma \in \Sigma_{l}^{n}$ such that $\psi_{\sigma}(T) \subseteq T^{\circ} \backslash \bigcup_{j=1}^{N}\left(K-e_{j}\right)$. Corollary 3.5 implies that $0=\boldsymbol{\mu}\left(\psi_{\sigma}(T)\right)=$ $W_{\sigma} \boldsymbol{\mu}(T)$, hence $W_{\sigma}=0$, a contradiction.

Remark. Let $\mathcal{F}:=\left\{\left(W_{\sigma} \mathbf{1}\right)^{\sim}: \sigma \in \Sigma_{l}^{*}\right\}$. Since each $\left(W_{\sigma} \mathbf{1}\right)^{\sim}$ is an $N$-vector with values 0 and $1, \mathcal{F}$ has cardinality $\leq 2^{N}$. Let $\mathcal{F}_{0}=\{\mathbf{1}\}$, $\mathcal{F}_{1}=\left\{\left(W_{j} \mathbf{1}\right)^{\sim}: j \in \Sigma_{l}\right\} \cup \mathcal{F}_{0}$ and

$$
\mathcal{F}_{n+2}=\mathcal{F}_{n+1} \cup\left\{\left(W_{i} \alpha\right)^{\sim}: i \in \Sigma_{l}, \alpha \in \mathcal{F}_{n+1} \backslash \mathcal{F}_{n}\right\}, \quad n \geq 0 .
$$

Note that (5.1) implies $\left(W_{\sigma \tau} \mathbf{1}\right)^{\sim}=\left(W_{\sigma}\left(W_{\tau} \mathbf{1}\right)^{\sim}\right)^{\sim}$, so $\left(W_{\tau} \mathbf{1}\right)^{\sim} \in \mathcal{F}_{n}$ if $|\tau|=$ $n$. Hence there exists an $n>0$ such that $\mathcal{F}_{n}=\mathcal{F}_{n+1}$ and, for this $n, \mathcal{F}=\mathcal{F}_{n}$. Theorem 5.1(iii) can be used to check whether the attractor $K$ has nonvoid interior in at most $2^{N}$ steps.

In the following we will use the above setup to consider the Lebesgue measure of a self-affine region. According to the Remark we denote the set of distinct elements of $\mathcal{F}=\left\{\left(W_{\sigma} \mathbf{1}\right)^{\sim}: \sigma \in \Sigma_{l}^{*}\right\}$ by $\left\{\mathbf{1}=v_{1}, v_{2}, \ldots, v_{r}\right\}$. Let $\alpha\left(v_{t}\right)$ denote the number of nonzero entries of $v_{t}$, and let

$$
N_{n, t}=\#\left\{\sigma \in \Sigma_{l}^{n}:\left(W_{\sigma} \mathbf{1}\right)^{\sim}=v_{t}\right\}, \quad n>0 .
$$

Let $\mathcal{B}_{n}$ be the tile partition defined in (3.3), and let

$$
\mathcal{B}_{n}^{*}=\left\{E \in \mathcal{B}_{n}: E^{\circ} \cap K \neq \emptyset\right\} .
$$

It is easy to see that $\bigcap_{n=1}^{\infty} \bigcup_{E \in \mathcal{B}_{n}^{*}} E=K$.

LEMMA 5.2. With the above notation, we have

(i) $\mathcal{L}(K)=\lim _{n \rightarrow \infty} l^{-n} \# \mathcal{B}_{n}^{*}$;

(ii) $\# \mathcal{B}_{n}^{*}=\sum_{t=1}^{r} N_{n, t} \alpha\left(v_{t}\right), n=1,2, \ldots$ 
Proof. Since $T$ is a $\mathbb{Z}^{d}$-tile, $\mathcal{L}(T)=1$. Therefore $\mathcal{L}(E)=l^{-n} \mathcal{L}(T)=l^{-n}$ for $E \in \mathcal{B}_{n}^{*}$, and

This proves (i).

$$
\mathcal{L}(K)=\lim _{n \rightarrow \infty} \mathcal{L}\left(\bigcup_{E \in \mathcal{B}_{n}^{*}} E\right)=\lim _{n \rightarrow \infty} \sum_{E \in \mathcal{B}_{n}^{*}} \mathcal{L}(E)=\lim _{n \rightarrow \infty} l^{-n} \# \mathcal{B}_{n}^{*} .
$$

For each $E \in \mathcal{B}_{n}$, there exist unique $\sigma \in \Sigma_{l}^{n}$ and $e_{t}$ such that $E=$ $\psi_{\sigma}(T)+e_{t}$ (Lemma 3.1) and $\boldsymbol{\mu}\left(\psi_{\sigma}(\partial T)\right)=0$ for all $\sigma \in \Sigma_{l}^{*}$ (Lemma 2.2). Hence Corollary 3.5 implies $\psi_{\sigma}(T)+e_{t} \in \mathcal{B}_{n}^{*}$ if and only if the $t$ th row of $W_{\sigma}$ is nonzero (i.e. the $t$ th coordinate of $\left(W_{\sigma} \mathbf{1}\right)^{\sim}$ is 1 ). This means that, for any given $\sigma \in \Sigma_{l}^{n}$, the number of nonzero rows of $W_{\sigma}$ is given by $\#\left\{\psi_{\sigma}(T)+e_{t} \in\right.$ $\left.\mathcal{B}_{n}^{*}: 1 \leq t \leq N\right\}=\mathbf{1}^{t}\left(W_{\sigma} \mathbf{1}\right)^{\sim}$. Hence

$$
\# \mathcal{B}_{n}^{*}=\sum_{\sigma \in \Sigma_{l}^{n}} \#\left\{\psi_{\sigma}(T)+e_{t} \in \mathcal{B}_{n}^{*}: 1 \leq t \leq N\right\}=\sum_{\sigma \in \Sigma_{l}^{n}} \mathbf{1}^{t}\left(W_{\sigma} \mathbf{1}\right)^{\sim} .
$$

The identity in (ii) follows directly from this and the definition of $N_{n, t}$.

Let $G$ be the $r \times r$ matrix defined by

$$
G(s, t)=l^{-1} \#\left\{i \in \Sigma_{l}:\left(W_{i} v_{s}\right)^{\sim}=v_{t}\right\}, \quad 1 \leq s, t \leq r,
$$

where $l$ is the number of $W_{i}$ in Theorem 1.1 and $\mathcal{F}=\left\{\mathbf{1}=v_{1}, v_{2}, \ldots, v_{r}\right\}$. It is clear from the definition that each row sum of $G$ is 1 , hence $G$ is a Markov matrix.

Before going on, we will recall some basic facts on the Perron-Frobenius theory on nonnegative matrices [1]. If a Markov matrix $B$ is primitive then it is easy to show that $\lim _{n \rightarrow \infty} B^{n}$ exists. For the Markov matrix $G$, there is $q>0$ and a permutation matrix $P$ such that

$$
P G^{q} P^{t}=\left[\begin{array}{cc}
X & Y \\
0 & Z
\end{array}\right] \text { with } Z=\left[\begin{array}{cccc}
R_{1} & 0 & \cdots & 0 \\
0 & R_{2} & \cdots & 0 \\
\vdots & & \ddots & \vdots \\
0 & 0 & \cdots & R_{k}
\end{array}\right] \text {, }
$$

where each $R_{i}$ is primitive with maximal eigenvalue $\varrho\left(R_{i}\right)=1$, and $X$ has maximal eigenvalue $\varrho(X)<1$.

It follows that $\lim _{n \rightarrow \infty} Z^{n}=R_{0}$ exists. Since $\varrho(X)<1, \lim _{n \rightarrow \infty} X^{n}=0$ and each $R_{i}$ is primitive. Therefore $\lim _{n \rightarrow \infty} G^{q n}=G_{0}^{(q)}$ exists and

$$
\begin{aligned}
P G_{0}^{(q)} P^{t} & =\lim _{n \rightarrow \infty} P G^{q n} P^{t} \\
& =\lim _{n \rightarrow \infty}\left[\begin{array}{cc}
X^{n} & X^{n-1} Y+X^{n-2} Y Z+\cdots+Y Z^{n-1} \\
0 & Z^{n}
\end{array}\right] \\
& =\left[\begin{array}{cc}
0 & (I-X)^{-1} Y R_{0} \\
0 & R_{0}
\end{array}\right] .
\end{aligned}
$$


If in addition $G$ has rational entries, we claim that the limiting matrix $G_{0}^{(q)}$ also has rational entries. Indeed, in view of (5.4), we can assume $G$ to be primitive. In that case, 1 is a simple eigenvalue and all the other eigenvalues have moduli $<1$. Let $\mathbf{u}$ be the left 1-eigenvector with $\sum_{j} u_{j}=1$. Then

$$
\lim _{n \rightarrow \infty} G^{n}=\left[\begin{array}{c}
\mathbf{u} \\
\vdots \\
\mathbf{u}
\end{array}\right] .
$$

Hence the claim will follow if we can show that $\mathbf{u}$ is rational. Note that if $G$ is of order $k$, then $G-I$ has rank $k-1$. We can assume

$$
G-I=\left[\begin{array}{ll}
C & * \\
\mathbf{b} & *
\end{array}\right],
$$

where $C$ is a $(k-1) \times(k-1)$ nonsingular matrix. It is checked directly that $\left[-\mathbf{b} C^{-1}, 1\right]$ is a left 1-eigenvector of $G$ by noticing that $G-I$ has rank $k-1$ and has rational coordinates. By uniqueness it equals $\mathbf{u}$ after normalization. This proves the claim.

TheOREM 5.3. Let $K$ be the attractor generated by the integral affine pair $(A, \mathcal{D})$. Let $G$ be defined as in (5.3). Then $\lim _{n \rightarrow \infty} G^{n q}$ exists for some $q$ and $\mathcal{L}(K)$ is rational. Furthermore,

$$
\mathcal{L}(K)=\sum_{j=1}^{r} a_{j} \alpha\left(v_{j}\right),
$$

where $\left[a_{1}, \ldots, a_{r}\right]$ is the first row of $G_{0}^{(q)}=\lim _{n \rightarrow \infty} G^{q n}$ given by (5.4).

Proof. First we claim that $G$ satisfies

$$
G^{n}(s, t)=l^{-n} \#\left\{\sigma \in \Sigma_{l}^{n}:\left(W_{\sigma} v_{s}\right)^{\sim}=v_{t}\right\}, \quad 1 \leq s, t \leq r .
$$

The case $n=1$ follows from the definition. Assume that (5.5) is true for $n>0$, and consider $G^{n+1}$. Since $\left(W_{i} W_{\sigma} \mathbf{1}\right)^{\sim}=\left(W_{i}\left(W_{\sigma} \mathbf{1}\right)^{\sim}\right)^{\sim}$ by (5.1), we have

$$
\begin{aligned}
G^{n+1}(s, t) & =\sum_{i=1}^{r} G(s, i) G^{n}(i, t) \\
& =l^{-n-1} \sum_{i=1}^{r} \#\left\{j \in \Sigma_{l}:\left(W_{j} v_{s}\right)^{\sim}=v_{i}\right\} \#\left\{\sigma \in \Sigma_{l}^{n}:\left(W_{\sigma} v_{i}\right)^{\sim}=v_{t}\right\} \\
& =l^{-n-1} \sum_{i=1}^{r} \#\left\{\sigma j \in \Sigma_{l}^{n+1}:\left(W_{j} v_{s}\right)^{\sim}=v_{i},\left(W_{\sigma} v_{i}\right)^{\sim}=v_{t}\right\} \\
& =l^{-n-1} \#\left\{\tau \in \Sigma_{l}^{n+1}:\left(W_{\tau} v_{s}\right)^{\sim}=v_{t}\right\},
\end{aligned}
$$

proving the claim. 
This implies that $N_{n, t}=l^{n} G^{n}(1, t), t=1, \ldots, r$. By Lemma 5.2,

$$
\mathcal{L}(K)=\lim _{n \rightarrow \infty} l^{-n} \# \mathcal{B}_{n}^{*}=\lim _{n \rightarrow \infty} \mathbf{e}_{1} G^{n} \alpha(v) .
$$

Now with the choice of $q$, it follows from the above digression on nonnegative matrices that $G_{0}^{(q)}=\lim _{n \rightarrow \infty} G^{q n}$ exists and $\mathcal{L}(K)$ has the expression as in the theorem follows. That $\mathcal{L}(K)$ is rational also follows from the digression.

We remark that the theorem and (5.4) allow us to obtain a simple algorithm to calculate $\mathcal{L}(K)$. That $\mathcal{L}(K)$ is rational was proved in [14] using a different method.

Next we consider the case of $K^{\circ}=\emptyset$. Theorem 5.1 implies that $W_{\sigma}=0$ for some $\sigma$. Without loss of generality, let $v_{r}=0$. Then $G$ has the following expression:

$$
G=\left[\begin{array}{cc}
G_{1} & g \\
0 & 1
\end{array}\right], \quad g \neq 0 .
$$

We denote the maximal eigenvalue of $G_{1}$ by $\lambda_{1}$.

LEMma 5.4. With the above notations, if $K^{\circ}=\emptyset$, we have

(i) $0<\lambda_{1}<1$;

(ii) $\lim _{n \rightarrow \infty} \frac{\log \# \mathcal{B}_{n}^{*}}{-\log \varrho^{n}}=d-\frac{\log \lambda_{1}}{\log \varrho}$, where $\varrho=|\operatorname{det} A|^{-1 / d}$.

Proof. (i) For any $1 \leq s \leq r-1$, assume that the $t$ th coordinate of $v_{s}$ is positive. Since $\sum_{j} W_{j}$ is irreducible, there exists $W_{j}$ such that the $t$ th column of $W_{j}$ is nonzero, so $W_{j} v_{s} \neq 0$. Hence the $s$ th row of $G_{1}$ contains at least one nonzero entry, which is $\geq l^{-1}$ by the definition of $G_{1}$. This means that each row sum of $G_{1}$ is at least $l^{-1}$, and therefore $\lambda_{1} \geq l^{-1}>0$.

By Theorem 5.1, there is a $\sigma \in \Sigma_{l}^{n}$ such that $W_{\sigma}=0$. (5.5) implies that all entries in the last column of $G^{n}$ are positive. This means that all row sums of $G_{1}^{n}$ are less than 1 . Hence $\lambda_{1}<1$.

(ii) By the definition of $N_{n, i},(5.5)$ implies $N_{n, s}=l^{n} G^{n}(1, s)$ for any $1 \leq s \leq r$. Using Lemma 5.2 (ii) and $\alpha\left(v_{r}\right)=0$, we have

$$
\# \mathcal{B}_{n}^{*}=l^{n}[1,0, \ldots, 0] G_{1}^{n} \alpha,
$$

where $\alpha=\left[\alpha\left(v_{1}\right), \ldots, \alpha\left(v_{r-1}\right)\right]^{t}$ is positive. Let $\beta$ be a nonnegative right $\lambda_{1}$-eigenvector of $G_{1}$ satisfying $\|\beta\|_{1}=1$. Then $\beta \leq \alpha$ coordinatewise. For any $1 \leq j \leq r-1$, let $\sigma \in \Sigma^{*}$ be such that $\left(W_{\sigma} \mathbf{1}\right)^{\sim}=v_{j}$. As (5.5) implies that there exists $k \geq 0$ such that the $(1, j)$ entry of $G_{1}^{k}$ is positive, we have $[1,0, \ldots, 0] G_{1}^{k} \beta=\lambda_{1}^{k}[1,0, \ldots, 0] \beta=\lambda_{1}^{k} \beta_{1}>0$. This implies that $\beta_{1}=$ $[1,0, \ldots, 0] \beta>0$. Hence $\# \mathcal{B}_{n}^{*} \geq l^{n}[1,0, \ldots, 0] G_{1}^{n} \beta=\varrho^{-n d} \lambda_{1}^{n} \beta_{1}>0$. It follows that

$$
\liminf _{n \rightarrow \infty} \frac{\log \# \mathcal{B}_{n}^{*}}{-\log \varrho^{n}} \geq d-\frac{\log \lambda_{1}}{\log \varrho} .
$$


On the other hand, for $\lambda>\lambda_{1}$, we have $\lambda^{-n} G_{1}^{n} \rightarrow 0$ as $n \rightarrow \infty$. There is a constant $a_{\lambda}>0$ such that $\# \mathcal{B}_{n}^{*}=l^{n}[1,0, \ldots, 0] G_{1}^{n} \alpha \leq l^{n} a_{\lambda} \lambda^{n}=\varrho^{-n d} a_{\lambda} \lambda^{n}$ $(n>0)$. Therefore

$$
\limsup _{n \rightarrow \infty} \frac{\log \# \mathcal{B}_{n}^{*}}{-\log \varrho^{n}} \leq d-\frac{\log \lambda}{\log \varrho}
$$

for any $\lambda>\lambda_{1}$, and (ii) follows by combining the estimations of the limsup and liminf.

THEOREM 5.5. Let $K$ be the attractor of an integral affine pair $(A, \mathcal{D})$ with $A$ a similarity. Suppose $K^{\circ}=\emptyset$. Then

$$
\operatorname{dim}_{\mathrm{B}} K=\operatorname{dim}_{\mathrm{H}} K=d-\log \lambda_{1} / \log \varrho<d .
$$

Proof. The theorem follows by showing that

$$
d-\log \lambda_{1} / \log \varrho \geq \overline{\operatorname{dim}}_{\mathrm{B}} K \geq \operatorname{dim}_{\mathrm{H}} K \geq d-\log \lambda_{1} / \log \varrho .
$$

For $E \subset \mathbb{R}^{d}$ and $\delta>0$, let $E_{\delta}=\left\{y \in \mathbb{R}^{d}:\|x-y\|<\delta\right.$ for some $\left.x \in E\right\}$ be the $\delta$-neighborhood of $E$. Let $\delta_{n}=\varrho^{n}$. It is clear that

$$
\mathcal{L}\left(E_{\delta_{n}}\right)=\varrho^{n d} \mathcal{L}\left(T_{1}\right) \quad \forall E \in \mathcal{B}_{n}^{*}, n>0 .
$$

(Here $T_{1}$ is the 1-neighborhood of $T$.) It follows from $K_{\delta_{n}} \subseteq \bigcup_{E \in \mathcal{B}_{n}^{*}} E_{\delta_{n}}$ that

$$
\mathcal{L}\left(K_{\delta_{n}}\right) \leq \sum_{E \in \mathcal{B}_{n}^{*}} \mathcal{L}\left(E_{\delta_{n}}\right)=\left(\# \mathcal{B}_{n}^{*}\right) \varrho^{n d} \mathcal{L}\left(T_{1}\right) .
$$

By Lemma 5.4(ii), we have

$$
\liminf _{n \rightarrow \infty} \frac{\log \mathcal{L}\left(K_{\delta_{n}}\right)}{\log \varrho} \geq \frac{\log \lambda_{1}}{\log \varrho} .
$$

Hence the first inequality in (5.6) holds in view of [8, Proposition 3.3.2].

The second inequality is well known. For the third, since $\left(T^{\circ}+e_{i}\right) \cap K \neq \emptyset$ for any $e_{i} \in \mathcal{E}$, we can find a constant $\varepsilon>0$ and points $x_{i} \in K$ such that $B_{\varepsilon}\left(x_{i}\right) \subseteq T^{\circ}+e_{i}, 1 \leq i \leq N$. Choose an invariant open set $V$ of the IFS $\left\{S_{i}\right\}_{i=1}^{m}$ such that $K \subset V$. Then there exists $k>0$ such that $\left|A^{-k}(V)\right|<\frac{1}{2} \varepsilon$. Hence there exists $I_{i} \in \Sigma_{m}^{k}$ such that

$$
S_{I_{i}}(\bar{V}) \subseteq B_{\varepsilon}\left(x_{i}\right) \subseteq T^{\circ}+e_{i}, \quad 1 \leq i \leq N .
$$

For any $E \in \mathcal{B}_{n}^{*}$, we can write $E=\psi_{\sigma}(T)+e_{u}=S_{I}\left(T+e_{i}\right)$ for some $\sigma \in \Sigma_{l}^{n}, I \in \Sigma_{m}^{n}$ and $e_{u}, e_{i} \in \mathcal{E}$ (by Lemma 3.1(iii) and the proof of Theorem 1.1). Hence $S_{I I_{i}}(\bar{V}) \subset E^{\circ}$. Therefore, there exist $I_{E} \in \Sigma_{m}^{n+k}$ such that $S_{I_{E}}(\bar{V}) \subset E$. Let $\Psi_{n}$ be the set of all those $S_{I_{E}}$; they are in one-to-one correspondence with $E \in \mathcal{B}_{n}^{*}$, so $\Psi_{n}$ has cardinality $\# \mathcal{B}_{n}^{*}$. We use this class of maps as an IFS; each $\Psi_{n}$ has contraction ratio $\varrho^{n+k}$, and from (5.7), they satisfy the open set condition. Let $K_{n}$ be the attractor. It follows from the well known identity that

$$
\operatorname{dim}_{\mathrm{H}} K_{n}=-\log \left(\# \mathcal{B}_{n}^{*}\right) /((n+k) \log \varrho) .
$$


Since $K_{n} \subseteq K$, we have $\operatorname{dim}_{\mathrm{H}} K \geq-\log \left(\# \mathcal{B}_{n}^{*}\right) /((n+k) \log \varrho)$ for all $n>0$. Hence Lemma 5.4 implies that the third inequality in (5.6) holds.

EXAmPle 5.6. Let $A=\left[\begin{array}{cc}2 & 1 \\ -1 & 2\end{array}\right], \mathcal{D}=\left\{[0,0]^{t},[1,0]^{t},[0,1]^{t},[1,1]^{t},[2,-1]^{t}\right\}$ and let $K$ be the attractor generated by $(A, \mathcal{D})$. Then $\operatorname{dim}_{\mathrm{H}} K \approx 1.820$.

For this we let

$$
\mathcal{C}=\left\{[0,0]^{t},[1,0]^{t},[0,1]^{t},[-1,0]^{t},[0,-1]^{t}\right\} .
$$

Then, from the remark after Lemma 2.1 , we see that $T=T(A, \mathcal{C})$ is a $\mathbb{Z}^{2}$-tile, and $T^{\circ} \cap K \neq 0$, since $\mathcal{C}=Q \cap \mathbb{Z}^{2}$. Let $\mathcal{E}_{1}=\left\{[0,0]^{t}\right\}$. By using Proposition 3.6 , we find $\mathcal{E}=\left\{[0,0]^{t},[1,0]^{t},[0,1]^{t},[1,1]^{t}\right\}$.

Let $p_{1}=p_{2}=p_{3}=p_{4}=p_{5}=1 / 5$. By the definition of $W_{i}$, we have

$$
\begin{aligned}
& W_{1}=\frac{1}{5}\left[\begin{array}{llll}
1 & 0 & 0 & 0 \\
1 & 0 & 0 & 0 \\
0 & 0 & 1 & 1 \\
0 & 0 & 0 & 0
\end{array}\right], \quad W_{2}=\frac{1}{5}\left[\begin{array}{llll}
1 & 1 & 0 & 0 \\
0 & 1 & 0 & 0 \\
0 & 0 & 0 & 1 \\
0 & 0 & 0 & 0
\end{array}\right], \quad W_{3}=\frac{1}{5}\left[\begin{array}{llll}
1 & 0 & 1 & 0 \\
0 & 1 & 1 & 0 \\
0 & 0 & 0 & 0 \\
0 & 0 & 0 & 0
\end{array}\right], \\
& W_{4}=\frac{1}{5}\left[\begin{array}{llll}
0 & 0 & 0 & 0 \\
0 & 0 & 0 & 0 \\
0 & 0 & 1 & 0 \\
0 & 1 & 0 & 1
\end{array}\right], \quad W_{5}=\frac{1}{5}\left[\begin{array}{llll}
0 & 0 & 0 & 0 \\
0 & 0 & 0 & 0 \\
1 & 1 & 1 & 1 \\
0 & 0 & 0 & 1
\end{array}\right] .
\end{aligned}
$$

Therefore

$$
\begin{aligned}
& \mathcal{F}_{1}=\left\{[1,1,1,1]^{t},[1,1,1,0]^{t},[0,0,1,1]^{t},[1,1,0,0]^{t}\right\}, \\
& \mathcal{F}_{2}=\mathcal{F}_{1} \cup\left\{[0,0,1,0]^{t}\right\}, \quad \mathcal{F}_{3}=\mathcal{F}_{2} \cup\left\{[0,0,0,1]^{t}\right\}, \\
& \mathcal{F}_{4}=\mathcal{F}_{3} \cup\left\{[0,0,0,0]^{t}\right\}=\mathcal{F}_{5} .
\end{aligned}
$$

Hence $\mathcal{F}=\mathcal{F}_{4}$ and $K^{0}=\emptyset$. It follows that

$$
G_{1}=\frac{1}{5}\left[\begin{array}{llllll}
0 & 2 & 2 & 1 & 0 & 0 \\
0 & 1 & 1 & 2 & 1 & 0 \\
0 & 0 & 2 & 1 & 2 & 0 \\
0 & 0 & 0 & 3 & 1 & 1 \\
0 & 0 & 0 & 1 & 3 & 0 \\
0 & 0 & 1 & 0 & 2 & 1
\end{array}\right],
$$

so that $\lambda_{1} \approx 0.882$ and $\operatorname{dim}_{\mathrm{H}} K=2-\log \lambda_{1} /(-\log 2) \approx 1.820$.

REMARK 5.7. When $A$ is not a similarity matrix, but all its eigenvalues have the same modulus, then by using a similar proof, we can show that the above theorem still holds. The reader is referred to [6] for details. 
6. The $L^{q}$-spectrum. Let $\left\{B_{\delta}\left(x_{i}\right)\right\}_{i}$ denote a family of disjoint balls with radius $\delta$ and centers $x_{i} \in K$. The $L^{q}$-spectrum (or moment scaling exponent) of a self-similar measure $\mu$ is defined by

$$
\tau(q)=\lim _{\delta \rightarrow 0+} \frac{\log \left(\sup \sum_{i} \mu\left(B_{\delta}\left(x_{i}\right)\right)^{q}\right)}{\log \delta}
$$

if the above limit exists, where the supremum is taken over all such families of balls [8]. (If the limit does not exist, one can replace the limit by lim inf.)

Proposition 6.1. Let $\mu$ be the self-similar measure generated by the integral similar pair $(A, \mathcal{D})$. Then

$$
\tau(q)=\lim _{n \rightarrow \infty} \frac{\log \sum_{E \in \mathcal{B}_{n}} \mu(E)^{q}}{n \log \varrho}, \quad q>0,
$$

where $\mathcal{B}_{n}=\left\{\psi_{\sigma}(T)+e: e \in \mathcal{E}, \sigma \in \Sigma_{q}^{n}\right\}$ is a tile-partition of $K$ defined in (3.3).

Proof. Let $a=1+|T|$ where $|T|$ is the diameter of $T$. From [31], we know that the limit in the definition of $\tau(q)$ exists for all $q \geq 0$. Hence it suffices to show that

$$
\sum_{E \in \mathcal{B}_{n}} \mu(E)^{q} \approx \sup \sum_{i} \mu\left(B_{a \varrho^{n}}\left(x_{i}\right)\right)^{q}, \quad q, n>0,
$$

where the supremum is taken over all families of disjoint balls $\left\{B_{a \varrho^{n}}\left(x_{i}\right)\right\}_{i}$ with $x_{i} \in K$. For such a family, let

$$
\mathcal{F}_{n, x_{i}}=\left\{E \in \mathcal{B}_{n}: E \cap B_{a \varrho^{n}}\left(x_{i}\right) \neq \emptyset\right\}, \quad \mathcal{G}_{n, E}=\left\{i: E \cap B_{a \varrho^{n}}\left(x_{i}\right) \neq \emptyset\right\} .
$$

It is easy to see that there exists a constant $b>0$ such that

$$
\max _{i} \# \mathcal{F}_{n, x_{i}}, \max _{E \in \mathcal{B}_{n}} \# \mathcal{G}_{n, E} \leq b \text {. }
$$

Hence

$$
\begin{aligned}
\sum_{i} \mu\left(B_{a \varrho^{n}}\left(x_{i}\right)\right)^{q} & \leq \sum_{i} \mu\left(\bigcup\left\{E \in \mathcal{F}_{n, x_{i}}\right\}\right)^{q} \\
& \leq \sum_{i} b^{q}\left(\max \left\{\mu(E): E \in \mathcal{F}_{n, x_{i}}\right\}\right)^{q} \\
& \leq b^{q+1} \sum\left\{\mu(E)^{q}: E \in \mathcal{B}_{n}\right\} \quad \forall q \geq 0 .
\end{aligned}
$$

It follows that

$$
\sup \sum_{i} \mu\left(B_{a \varrho^{n}}\left(x_{i}\right)\right)^{q} \leq b^{q+1} \sum_{E \in \mathcal{B}_{n}} \mu(E)^{q} \quad \forall q \geq 0 .
$$

On the other hand, for each $E \in \mathcal{B}_{n}$ satisfying $\mu(E)>0$, choose a point from $K \cap E$ and denote this set by $\left\{y_{i}: i=1, \ldots, r\right\}$. Then we have

$$
\sum_{E \in \mathcal{B}_{n}} \mu(E)^{q} \leq \sum_{i=1}^{r} \mu\left(B_{a \varrho^{n}}\left(y_{i}\right)\right)^{q} \quad \forall q \geq 0, n>0 .
$$


For the family $\left\{B_{a \varrho^{n}}\left(y_{i}\right)\right\}$, we can choose a disjoint subfamily $\left\{B_{a \varrho^{n}}\left(y_{i_{j}}\right)\right\}$ and a number $s$ depending only on $T$ and $d$ such that:

(i) $\#\left\{i: B_{a \varrho^{n}}\left(y_{i}\right) \cap B_{a \varrho^{n}}\left(y_{i_{j}}\right) \neq \emptyset\right\} \leq s$ for all $i_{j}$ (note that $B_{a \varrho^{n}}\left(y_{i}\right) \cap$ $B_{a \varrho^{n}}\left(y_{i_{j}}\right) \neq \emptyset$ implies $\left.E_{i} \subseteq B_{2 a \varrho^{n}}\left(y_{i_{j}}\right)\right)$;

(ii) $\mu\left(B_{a \varrho^{n}}\left(y_{i_{1}}\right)\right)=\max _{i \geq 1} \mu\left(B_{a \varrho^{n}}\left(y_{i}\right)\right)$ and for $j \geq 2, \mu\left(B_{a \varrho^{n}}\left(y_{i_{j}}\right)\right)=$ $\max \left\{\mu\left(B_{a \varrho^{n}}\left(y_{i}\right)\right): B_{a \varrho^{n}}\left(y_{i}\right) \cap \bigcup_{k=1}^{j-1} B_{a \varrho^{n}}\left(y_{i_{k}}\right)=\emptyset\right\} ;$

(iii) any $B_{a \varrho^{n}}\left(y_{i}\right)$ intersects at least one $B_{a \varrho^{n}}\left(y_{i_{j}}\right)$.

Therefore (6.4) implies

$$
\sum_{E \in \mathcal{B}_{n}} \mu(E)^{q} \leq s \sum_{j} \mu\left(B_{a \varrho^{n}}\left(y_{i_{j}}\right)\right)^{q} \leq s \sup \sum_{i} \mu\left(B_{a \varrho^{n}}\left(x_{i}\right)\right)^{q} \quad \forall q \geq 0
$$

(the second inequality is by (i)), and (6.2) follows from (6.3) and (6.5).

We can now express $\tau(q)$ in terms of the transition matrices $\left\{W_{i}\right\}_{j=1}^{l}$ in Theorem 1.1.

THEOREM 6.2. Let $\mu$ be the self-similar measure generated by the integral similar pair $(A, \mathcal{D})$. Then

$$
\tau(q)=\lim _{n \rightarrow \infty} \frac{\log \sum_{\sigma \in \Sigma_{l}^{n}}\left\|W_{\sigma}\right\|_{1}^{q}}{n \log \varrho}, \quad q \geq 0,
$$

where $W_{\sigma}$ is defined in Theorem 1.1, and $\left\|W_{\sigma}\right\|_{1}$ is the sum of all entries of $W_{\sigma}$.

Proof. Let $\mathbf{e}_{i}$ be the $i$ th column of the $N \times N$ identity matrix. From Lemma 3.1(iii), Lemma 3.3 and Corollary 3.5, for all $n>0$ we have

$$
\sum_{E \in \mathcal{B}_{n}} \mu(E)^{q}=\sum_{r=1}^{N} \sum_{\sigma \in \Sigma_{l}^{n}} \mu\left(A^{-n}\left(T+c_{\sigma}\right)+e_{r}\right)^{q}=\sum_{r=1}^{N} \sum_{\sigma \in \Sigma_{l}^{n}}\left(\mathbf{e}_{r}^{t} W_{\sigma} \boldsymbol{\mu}(T)\right)^{q} .
$$

Using $\left(\sum_{i=1}^{N} a_{i}\right)^{q} \approx \sum_{i=1}^{N} a_{i}^{q} \quad\left(N, q>0\right.$ fixed) for any $a_{i} \geq 0$, we have

$$
\sum_{r=1}^{N} \sum_{\sigma \in \Sigma_{l}^{n}}\left(\mathbf{e}_{r}^{t} W_{\sigma} \boldsymbol{\mu}(T)\right)^{q} \approx \sum_{\sigma \in \Sigma_{l}^{n}}\left(\sum_{r=1}^{N} \mathbf{e}_{r}^{t} W_{\sigma} \boldsymbol{\mu}(T)\right)^{q} .
$$

Therefore

$$
\begin{aligned}
\tau(q) & =\lim _{n \rightarrow \infty} \frac{\log \sum_{\sigma \in \Sigma_{l}^{n}}\left(\sum_{r=1}^{N} \mathbf{e}_{r}^{t} W_{\sigma} \boldsymbol{\mu}(T)\right)^{q}}{n \log \varrho} \\
& =\lim _{n \rightarrow \infty} \frac{\log \sum_{\sigma \in \Sigma_{l}^{n}}\left\|W_{\sigma}\right\|_{1}^{q}}{n \log \varrho}, \quad q \geq 0,
\end{aligned}
$$

by using the fact that $\sum_{i=1}^{N} \mathbf{e}_{i}^{t}=[1, \ldots, 1]$ and $\boldsymbol{\mu}(T)$ is a fixed vector with strictly positive coordinates. 
Note that $\tau(q)$ is a concave function. For a concave function $g$ on $\mathbb{R}$, the Legendre transform (or concave conjugate) of $g$ is defined as

$$
g^{*}(\alpha)=\inf \{q \alpha-g(q): q \in \mathbb{R}\} .
$$

If $g$ is differentiable at $q$ and $g^{\prime}(q)=\alpha$, then $g^{*}(\alpha)=q \alpha-g(q)$.

For a Borel measure $\mu$ with support $K$, we let

$$
\alpha(x)=\lim _{r \rightarrow 0} \frac{\log \mu\left(B_{r}(x)\right)}{\log r}
$$

be the local dimension of $\mu$ at $x$. Let $K_{\alpha}=\{x \in K: \alpha(x)=\alpha\}$ be the $\alpha$-level set of $\mu$. A heuristic principle called multifractal formalism suggests that the dimension spectrum $\operatorname{dim}_{\mathrm{H}} K_{\alpha}$ should equal the Legendre transform of $\tau(q)$, i.e.,

$$
\tau^{*}(\alpha)=\operatorname{dim}_{\mathrm{H}} K_{\alpha} .
$$

This is the case when the IFS satisfies the OSC ([2], [24]). In the present case, by Theorem 1.1, $\sum_{i=1}^{l} W_{i}$ is irreducible, hence [11, Theorem 1.3] shows that $\tau(q)$ is differentiable for all $q>0$. Also the IFS satisfies the weak separation condition under our assumption of integral entries in $A$ and $\mathcal{D}$. Hence [18, Theorem B] implies that the multifractal formalism holds for all $q>0$ :

Theorem 6.3. Let $A$ be an integral similarity matrix. Let $\mu$ be the selfsimilar measure generated by the integral pair $(A, \mathcal{D})$. Then the $L^{q}$-spectrum $\tau(q)$ of $\mu$ is differentiable for all $q>0$ and

$$
\tau^{*}(\alpha)=\operatorname{dim}_{\mathrm{H}} K_{\alpha}, \quad \forall \alpha=\tau^{\prime}(q), q>0 .
$$

We do not have a complete understanding for $q<0$. In [25], it is shown that for some special cases the equality of Theorem 6.3 also holds for $q<0$. Note that there is a simple example where $\tau(q), q<0$, is not differentiable at one point: $A=3, \mathcal{D}=\{0,1,2,3\}$ and weights $\{1 / 8,3 / 8,3 / 8,1 / 8\}[28]$; there is a modification of the multifractal formalism for that case [12]. Other interesting cases were considered in [13], [38] and [34].

\section{References}

[1] A. Berman and R. J. Plemmons, Nonnegative Matrices in Mathematical Sciences, SIAM, 1994.

[2] R. Cawley and R. Mauldin, Multifractal decompositions of Moran fractals, Adv. Math. 92 (1992), 196-236.

[3] I. Daubechies and J. Lagarias, Two-scale difference equations I. Existence and global regularity of solutions, SIAM J. Math. Anal. 22 (1991), 1388-1410.

[4] - - - Two-scale difference equations II. Local regularity, infinite products of matrices and fractals, ibid. 23 (1992), 1031-1079.

[5] —, - Thermodynamic formalism for multifractal functions, Rev. Math. Phys. 6 (1994), 1033-1070. 
[6] Q. R. Deng, Iteration function systems with overlaps and self-affine measures, Ph.D. thesis, CUHK, 2005.

[7] G. Edgar and R. Mauldin, Multifractal decomposition of digraph recursive fractals, Proc. London Math. Soc. 65 (1992), 604-628.

[8] K. Falconer, Fractal Geometry-Mathematical Foundations and Applications, Wiley, 1990.

[9] D. Feng, The smoothness of $L^{q}$-spectrum of self-similar measures with overlaps, J. London Math. Soc. 68 (2003), 102-118.

[10] - , The limit Rademacher functions and Bernoulli convolution associated with Pisot numbers, Adv. Math. 195 (2005), 24-101.

[11] D. Feng and K. S. Lau, The pressure function for products of non-negative matrices, Math. Res. Lett. 9 (2002), 363-378.

[12] D. Feng, K. S. Lau and X. Y. Wang, Some exceptional phenomena in multifractal fomalism: Part II, Asian J. Math. 9 (2005), 473-488.

[13] D. Feng and Y. Wang, A class of self-affine measures, J. Fourier Anal. Appl. 11 (2005), 107-124.

[14] X. G. He, K. S. Lau and H. Rao, Self-affine sets and graph-directed systems, Constr. Approx. 19 (2003), 373-397.

[15] J. Hutchinson, Fractals and self-similarity, Indiana Univ. Math. J. 30 (1981), 713747.

[16] R. Q. Jia, Subdivision schemes in $L^{p}$ spaces, Adv. Comput. Math. 3 (1995), 309-341.

[17] R. Q. Jia, K. S. Lau and D. X. Zhou, $L_{p}$ solutions of refinement equations, J. Fourier Anal. Appl. 7 (2001), 143-167.

[18] J. Lagarias and Y. Wang, The finiteness conjecture for the generalized spectral radius of a set of matrices, Linear Algebra Appl. 214 (1995), 17-42.

[19] -, 一, Self-affine tiles in $\mathbb{R}^{n}$, Adv. Math. 121 (1996), 21-49.

[20] - - - Haar bases for $L^{2}\left(\mathbb{R}^{n}\right)$ and algebraic number theory, J. Number Theory 57 (1996), 181-197; Corrigendum/addendum, 76 (1999), 330-336.

[21] - - - Integral self-affine tiles in $\mathbb{R}^{n}$. I. Standard and nonstandard digit sets, J. London Math. Soc. (2) 54 (1996), 161-179.

[22] - - - Integral self-affine tiles in $\mathbb{R}^{n}$. II. Lattice tilings, J. Fourier Anal. Appl. 3 (1997), 83-102.

[23] K. S. Lau and S. M. Ngai, $L^{q}$-spectrum of the Bernoulli convolution associated with the golden ratio, Studia Math. 131 (1998), 225-251.

[24] -, -, Multifractal measures and a weak separation condition, Adv. Math. 141 (1999), 45-96.

[25] - - - Second order self-similar identities and multifractal decompositions, Indiana Univ. Math. J. 49 (2000), 925-972.

[26] K. S. Lau, S. M. Ngai and H. Rao, Iteration function systems with overlaps and self-similar measures, J. London Math. Soc. 63 (2001), 99-116.

[27] K. S. Lau and J. R. Wang, Characterization of $L^{p}$-solutions for the two-scale dilation equations, SIAM J. Math. Anal. 26 (1995), 1018-1046.

[28] K. S. Lau and X. Y. Wang, Some exceptional phenomena in multifractal formalism: Part I, Asian J. Math. 9 (2005), 275-294.

[29] R. Mauldin and S. Williams, Hausdorff dimension in graph directed constructions, Trans. Amer. Math. Soc. 309 (1988), 811-829.

[30] S. M. Ngai and Y. Wang, Hausdorff dimension of self-similar sets with overlaps, J. London Math. Soc. 63 (2001), 655-672.

[31] Y. Peres and B. Solomyak, Existence of $L^{q}$ dimensions and entropy dimension for self-conformal measures, Indiana Univ. Math. J. 49 (2000), 1603-1621. 
[32] A. Potiopa, A problem of Lagarias and Wang, in: Progr. Probab. 46, Birkhäuser, 2000, 39-65.

[33] H. Rao and Z. Y. Wen, A class of self-similar fractals with overlap structure, Adv. Appl. Math. 20 (1998), 50-72.

[34] P. Shmerkin, A modified multifractal formalism for a class of self-similar measures with overlap, Asian J. Math. 9 (2005), 323-348.

[35] B. Solomyak, On the random series $\sum \pm \lambda^{n}$ (an Erdös problem), Ann. of Math. 142 (1995), 611-625.

[36] R. Strichartz, Self-similar measures and their Fourier transforms III, Indiana Univ. Math. J. 42 (1993), 367-411.

[37] R. Strichartz and Y. Wang, Geometry of self-affine tiles, I, ibid. 48 (1999), 1-23.

[38] B. Testud, Étude d'une classe de mesures autosimilaires : calculs de dimensions et analyse multifractale, Ph.D. thesis, Univ. Blaise Pascal, 2004.

[39] A. Vince, Replicating tessellations, SIAM J. Discrete Math. 6 (1993), 501-521.

Department of Mathematics

Fujian Normal University

Fuzhou 350007, China

E-mail: qrdeng@fjnu.edu.cn

Department of Mathematics

The Chinese University of Hong Kong

Hong Kong

E-mail: kslau@math.cuhk.edu.hk
Department of Mathematics Central China Normal University

Wuhan 430079, China

E-mail: xingganghe@sina.com

Received November 30, 2007

Revised version May 15, 2008 